\title{
Microfibril Organization Modes in Plant Cell Walls of Variable Curvature: A Model System for Two Dimensional Anisotropic Soft Matter
}

\author{
${ }^{1}$ Department of Chemical Engineering \\ McGill University \\ 3610 University Street \\ Montreal, Quebec \\ Canada H3A 2B2 \\ ${ }^{2}$ Department of Mechanical Engineering \\ McGill University \\ 817 Sherbrooke West \\ Montreal, Quebec \\ Canada H3A $2 \mathrm{~K} 6$ \\ * corresponding author
}

Yogesh K. Murugesan ${ }^{1}$, Damiano Pasini ${ }^{2}$, and Alejandro D. Rey ${ }^{1^{*}}$

Submitted to: Soft Matter

Date: 3 May, 2011

Type: Journal article e-mail: alejandro.rey@ mcgill.ca

Tel: (514) 398-4196

Fax: (514) 398-6678

Keywords: Curvature-induced planar self assembly, 2D anisotropic soft matter, plant cell walls 


\section{Abstract}

We present a mechanical model of the plant cell wall viewed as anisotropic two dimensional soft matter, where a dilute dispersion of cellulose fibrils of variable orientations are uniformly distributed on a curved deformable viscoelastic matrix membrane. The plant cell wall model integrates the elastic energy of the curved membrane, the nematic Landau-de Gennes fiber orientation energy, and competing curvophilic and curvophobic interactions mediated by the membrane geometry and the fibrils' orientation. The selected membrane geometry is a straight cylinder of variable cross-section, whose shape varies from a circle to a sharp super-ellipse as in many cell specimens, and whose size increases as in plant cell wall growth. Model predictions indicate that due to curvature-orientation couplings, the fiber orientation display three modes: (i) line (along the cylinder axis), (ii) helical (oblique to the axis), and (iii) ring (normal to the axis), where the former arises under large curvature and the latter under small one. In addition to aligning the fibers in the line mode, high curvature also promotes the order of the fibrils. The predicted fiber structure is validated with fiber structures in the cell wall of tracheids. The structure-properties relations for super-elliptical membranes with gradient curvature are established and its role in cell growth shape is predicted. The principal mechanisms are the role of fiber orientation and order on bending stiffness: (a) orientation along the axis has no stiffening effect, (b) orientation along the azimuthal direction produces maximal stiffening, and (c) fiber randomization softens the membrane. The largest fiber stiffening effect is found when the membrane length scale (radius of curvature) and fiber length scale (curvophobic/curvophilic energy ratio) are equal. It is found that super-elliptical shape invariant growth and expansion is preferred for cells with sharp/ soft corners and straight/stiff sides. Otherwise growth promotes shape changes. Lastly, master plots that categorize fiber structures by the number of multimodal 
regimes (line/helix, line/helix/ring) that arise in super-ellipses, are used to relate shape and size to domain structures that affect toughness and modulus.

\section{Introduction}

Two dimensional anisotropic soft matter exhibits a combination of long range in-plane orientational order of filament inclusions and deformable membranes (or interfaces), that interact through novel surface phenomena such as curvophobic and curvophilic effects. Examples of these materials include: (a) liquid crystal interfaces and films ${ }^{1}$ of interest to the display industry ${ }^{2}$ and online stress field measurements ${ }^{3}$ due to their optical properties, (b) nematic shells of interest to biotechnological and pharmaceutical researchers owing to their structural similarity to vesicles ${ }^{4}$, and (c) graphite foams for novel heat transfer applications in electrical devices ${ }^{5}$. In addition, biological structural and functional materials including fiber-laden membranes such as plant cell walls $^{6}$ and protein containing lipid-bilayers ${ }^{7}$, are 2D anisotropic membranes of interest to researchers in biophysics and biomimetic materials development. Two dimensional self assembly and orientational order of rods, fibers, and filaments on soft deformable thin substrates remains partially understood despite its biological and biomimetic relevance. In this work, the delicate interplay between membrane geometry and embedded fiber order in biological fiber-laden membranes is explored using a model that integrates membrane elasticity and liquid crystal fiber ordering.

Biological fiber-laden membranes are conveniently classified as:

a) Hard fiber/soft membrane: the orientation of the fibrous fillers is fixed and the membrane adapts to the fiber structure by modifying its shape. A class of proteins called membrane-curving proteins such as clathrin adaptor protein complexes and BAR domain proteins 
that provide scaffolds for spherical and cylindrical membrane curvature are a well-known example of this case ${ }^{8}$;

b) Hard membrane/soft fiber: the membrane shape and size are fixed and the fiber orientation adapts to the membrane geometry by changing its conformation. An example of this case is antimicrobial peptides adsorbed onto microbial cell membrane resulting in cell lysis. It has been reported that the effectiveness of antimicrobial activity of these peptides depends strongly on concentration, orientation and degree of insertion of these peptide filaments onto the membrane ${ }^{7}$;

c) Soft fiber/soft membrane: the membrane shape and the fiber orientation are coupled and hence adapt to each other dynamically by simultaneously varying fiber structure and membrane shape. The plant cell wall, a structural element in the order of nanometers that surrounds every cell of a plant is an example of this class. This generic class reduces to case (a) when the fiber orientation is fixed, and case (b) when the membrane geometry is fixed. The generic class (c) is well represented by the plant cell wall and is the focus of this paper.

The plant cell wall is a multifunctional, dynamical structure that is made of sequentially deposited layers of different thickness, chemical constituents and composition, and structural organization?. The primary cell wall is the first layer to be laid down after cell division and its formation coincides with cell growth'. When the cell reaches its final size, three layers of secondary cell wall are laid over the primary cell wall in the inward direction; see Figure 3 below for details. The central layer, called secondary layer $2\left(\mathrm{~S}_{2}\right)$, comprises about $80-90 \%$ by volume of the entire cell wall. Towards the lumen (a cavity left by the cell after shrinkage and death of the cell) is the secondary layer $3\left(S_{3}\right)$. At the other side of $S_{2}$, there are the secondary layer $1\left(S_{1}\right)$ and the primary cell wall ${ }^{9}$. Growing cells are surrounded by primary cell wall and lack secondary cell wall layers. The primary cell wall has the following functions: it supports the cell membrane 
against the turgor pressure contained within the cell; it undergoes an enzyme controlled expansion under turgor pressure and regulates the direction of expansion contributing to growth and form of a plant; it cooperates with adjacent cells under turgor pressure to build a three-dimensional tissue. The secondary cell walls provide additional mechanical strength to the tissue and in some seeds perform energy storage. The shape of these membranes depends on the types of the cells. For example, compression wood cells of spruce are cylinders of circular or elliptical cross-section, while normal wood cells of spruce are often cylinders with super-elliptical cross-section ${ }^{\mathbf{1 0}}$.

The plant cell wall can be considered as a reinforced biological membrane consisting of cellulose microfibrils of high tensile strength embedded in a matrix of polysaccharides and structural glycoproteins 9 . These cellulose microfibrils in the extracellular matrix are oriented in strategic directions to generate commonly observed textures such as line, ring, helix, crossed helix and helicoid ${ }^{\mathbf{1 1}}$. The orientation of cellulose microfibrils in secondary cell wall layers governs the physical properties of wood and that in primary cell wall controls the shape of the cell and contributes to the morphology at the tissue and organ level12. The mechanisms by which the microfibrils are orientated in a specific direction are not well understood ${ }^{\mathbf{1 3}}$. In an earlier attempt to understand this mechanism, a mechanical model based on force balance on an open fiber reinforced cylinder representing reinforced biological membranes concluded that the fiber orientation angle with respect to the long axis of the cylinder should be $54.7^{\circ}$ at maximum volume of the cylinder and the volume of the cylinder will diminish towards zero at lower and higher fiber angles ${ }^{\mathbf{1 4}}$. For a closed cylindrical membrane of constant volume, the system can change its crosssectional shape from a circle to an ellipse depending on the system volume, while maintaining the fiber orientation angle with respect to the long axis of the cylinder at $54.7^{\circ}$. This theoretical 
prediction is in contradiction with experimentally observed microfibril angle in plant cell walls ${ }^{\mathbf{1 3}}$ that can range from $0^{\circ}$ to $90^{\circ}$.

In a later mesogenic self-assembly model, based on the observed structural similarity between the helicoidal texture and chiral nematic liquid crystals and defect patterns characteristic of chiral self-assembly observed in secondary cell wall of some plant species, it has been hypothesized that these structures arise through liquid crystalline self assembly ${ }^{15}$. According to this hypothesis, when the concentration of the microfibrils exceed Onsager's critical fiber concentration threshold, the interaction between these microfibrils result in the alignment of microfibrils in a specific direction as an attempt to minimize the excluded volume of the microfibril ${ }^{16}$. A review supporting this hypothesis by analyzing it from the aspects of anatomy and developmental biology has emphasized on the necessity of characterizing the nature of the forces inducing this self-assembly ${ }^{17}$. It is also known that the cellulose microfibrils extracted from plant cell walls by acid-hydrolysis self-assemble to form chiral-nematic phases in vitro ${ }^{18,19}$. Despite scant in-vivo experimental evidence and/or data, this hypothesis is strongly supported by theory and simulations based on well established liquid crystal theory. This is evident from the recent review that emphasizes the continuum Landau-de Gennes theory that yields testable and verifiable predictions of thermodynamical, textural, and rheological phenomena observed in biological systems $^{20}$. For an extensive review of mesoscopic models employed to quantitatively describe biological liquid crystalline phases and processes see reference [20]. Recently ${ }^{21}$, a mathematical model based on the Landau-de Gennes theory of liquid crystals has been used to simulate defect textures arising in the domain of self assembly due to the presence of secondary phases. This study concludes that the defect patterns observed in some plant cell walls are those expected from a truly liquid crystalline self-assembly process, supporting the above hypothesis ${ }^{21}$. 
In the present work, we use theory and simulation to investigate the possibility that the fiber cellulose microfibril orientation is imparted by the interaction between membrane curvature and embedded fiber order, when cellulose microfibril concentrations is less than that of Onsager's critical limit, and partially validate the model predictions through available experimental observations. An example of the possible effect of membrane curvature on microfibril orientation in plant cell walls is found in the abrupt changes in microfibril angle in the $S_{2}$ and $S_{3}$ layers at the corners of juniper tracheid plant cells ${ }^{\mathbf{1 4}}$. The existence of membrane curvature-driven fiber orientation is further supported by the transient nature of helicoidal pattern in primary plant cell walls of mung bean, owing to drastic change in cell curvature during the deposition of primary cell wall $^{22}$.

In an earlier attempt to estimate the influence of membrane curvature on embedded fibers, a theoretical model based on continuum elasticity theory for anisotropic membrane inclusions in lipid membranes was developed ${ }^{\mathbf{2 3}}$. The model neglected interactions between the inclusions and was able to predict line and ring orientation modes ${ }^{\mathbf{2 3}}$. Recently ${ }^{\mathbf{2 4}}$, an integrated mechanical model describing nematic liquid crystalline self-assembly of rigid rods on an arbitrarily curved membrane has been presented and relative predictions of cellulose ordering and orientation in the plant cell wall are presented. In this model ${ }^{24}$, the mechanics of the fluid membrane is described by the Helfrich bending-torsion membrane model, the fiber self-assembly is described by the 2D Landaude Gennes quadrupolar Q-tensor order parameter model, and the fiber-membrane interactions (inspired by an extension of the 2D Maier-Saupe model to curved surfaces) include competing curvophilic (curvature-seeking) and curvophobic (curvature-avoiding) effects. An integrated shape and nematic order equation developed in this work $^{\mathbf{2 4}}$ gives a complete model whose solution describes the coupled membrane shape and fiber order state. The analytical solution of the model 
for a cylindrical membrane of circular cross-section can predict line, ring and helical modes observed in some plant cell walls ${ }^{24}$. The model when integrated with the Gibbs- Duhem equation can describe the role of temperature and adsorption on membrane shape and fiber $\operatorname{order}^{25}$. A viscoelastic model that integrates the statics of anisotropic membranes developed in this model with the planar nematodynamics of fibers and the dynamics of isotropic membranes has been developed to study dynamic remodeling of plant cell wall during growth and morphogenesis ${ }^{26}$.

The main issue considered in this paper is self-assembly of rigid fibers representing microfibrils on a soft deformable non-planar 2D membrane . The membrane average (deviatoric) curvature is $\mathrm{H}(\mathrm{D})^{27,28}$; see section 2.1 . The fiber orientation is defined by the director $\mathbf{n}$ and exists when the scalar order parameter $\mathrm{S}$ is non-zero; see section 2.2. We consider a flat membrane $(\mathrm{H}=\mathrm{D}=0)$ with a random assembly of rigid rod fibers of length $\mathrm{L}$ and diameter $\mathrm{d}_{\mathrm{f}}$. When the fiber volume fraction $\varphi$ is such that $\varphi \mathrm{L}_{\mathrm{p}} / \mathrm{d}_{\mathrm{eff}}<\mathrm{C}$, which according to Onsager 3D model gives $\mathrm{C} \approx 4$, the fibers on a flat surface should be in the isotropic state ${ }^{29,30,31}(S=0) ; L_{p}$ is the fiber persistence length and $d_{\text {eff }}$ is the effective diameter of the fiber. The phenomena we wish to describe is how under sufficient curvature $(H \neq 0, D \neq 0)$, a $2 \mathrm{D}$ nematic state with a specific director orientation $\mathbf{n}$ and non-zero scalar order parameter S may arise through curvature-mediated interactions. The main concept is that introducing mechanical bending and torsion to the membrane creates a curvature field that then may generate a $2 \mathrm{D}$ nematic ordering with a particular director orientation. The $2 \mathrm{D}$ soft deformable, fluid membrane under consideration in this paper is described by the Helfrich model $^{32}$, and contains bending and torsion elasticity. The fibers interact with each other through excluded volume. The fiber and membrane interactions are mediated through the membrane curvature, such that both curvophilic and curvophobic effects are included; in the former (latter) $\mathbf{n}$ seeks to align along high (zero) curvature directions ${ }^{33}$. Thus the process to be described is the 
coupling of planar nematic liquid crystal self-assembly and membrane shape selection under the action of a pressure differential. Curvature-mediated interactions have been previously discussed in the literature ${ }^{33,34}$.

The second important motivation of this paper is the understanding and characterization of spatial heterogeneities in fiber-filled membranes. Since many biological and synthetic membranes have variable curvature $\left(\nabla_{\mathrm{s}} \mathrm{H} \neq 0, \nabla_{\mathrm{s}} \mathrm{D} \neq 0\right)$, the fiber response to curvature gradients is likely to produce heterogeneous fiber distributions. Restricting this comment to cylinders $(\mathrm{H}=\mathrm{D}$, the relation between fiber orientation and membrane curvature $\mathbf{n}=\mathbf{n}(\mathrm{H})$ leads to the expectation that smooth curvature gradients $\nabla_{\mathrm{s}} \mathrm{H}$ will result in fiber orientation gradients. For dilute fiber system, where gradient elasticity is small or insignificant, the fiber orientation gradients will be sharp. Hence it is expected that smooth curvature variations may lead to orientation domains along the membrane. Similar considerations apply to the order $S$ of the fibers.

The third motivation of this paper is the quantitative characterization of mechanical bending stiffness of membranes partitioned by domains of different fiber orientation and fiber order. In this 2D analysis, the bending of the membrane refers to changes in curvature in the crosssectional plane of the cylindrical membrane and not along the cylinder axis, resulting in crosssectional shape change while preserving its cylindrical shape. It is expected that curvature gradients that lead to orientation domains will results in gradients in the membrane bending modulus. For example, when the fibers are aligned along a cylinder axis, no stiffening effect is expected. On the other hand, when the fibers are along the azimuthal direction, a maximum stiffness is expected. In this paper the integration of these three motivations:

membrane curvature gradients $\Rightarrow$ fiber organization gradients $\Rightarrow$ bending stifness gradients 
is pursued in the context of cellulose-filled plant cell membranes, where it is assumed that fibers adapt and respond curvature. Once the bending stiffness profile is known, shape changes during growth can be predicted, as stiffer section will not bend to accommodate expansion. In partial summary, the specific objective of this work is to investigate the curvature-induced microfibril orientation in plant cell walls by solving an integrated micromechanical model developed for fiberladen membranes. The knowledge gained is then extended to cylindrical membranes of superelliptical cross-section (relevant to plant cell walls) in which the membrane curvature varies smoothly around the circumference of the membrane resulting in spatially graded fiber orientation.

The organization of this article is as follows. Section 2 presents the characterization of membrane geometry, the Lame' curves used to characterize the super-elliptical membrane crosssection, the 2D quadrupolar nematic order parameters $\mathbf{Q}$ used to characterize the fiber structure and the effective bending modulus used to characterize the bending stiffness of fiber-laden membranes. The average $\mathrm{H}$ and deviatoric $\mathrm{D}$ membrane curvatures used to characterize the membrane shape in this paper is derived in appendix A. Section 3 introduces the Helmholtz free energy density of the membranes, the 2D Landau-de Gennes nematic liquid crystal homogeneous energy of the planar fiber interactions, and the membrane-fiber coupling energies. The fiber structure plot depicting the fiber orientation regimes and their transitions is presented and validated. The Helmholtz free energy density in terms of fiber angle $\alpha$, fiber order $S$, and membrane curvatures H/D is derived and minimized to obtain the preferred fiber orientation and ordering modes in appendix B. The role of fiber structure on effective bending modulus of the fiber-laden membranes is also presented. The fiber and membrane contributions to effective bending modulus of the fiber-laden membranes is derived in appendix C. Section 4 utilizes the validated fiber structure plot to investigate fiber orientation modes for a set of super-elliptical 
membrane of variable curvature around the circumference of the membrane. Phase maps for fiber orientation modes and uniformity of fiber orientation distribution are also presented. The effective bending modulus plots presenting typical elastic inhomogeneities arising in super-elliptical membranes systems and their characteristic deformation mode are discussed. Section 5 presents the conclusions.

\section{Geometry, order parameters and elasticity}

\subsection{Membrane shape characterization}

In this paper, the geometry of an arbitrarily curved membrane is characterized by the curvature tensor $\mathbf{b}$ which can be decomposed into a trace and a deviatoric curvature tensor: $\mathbf{b}=\mathrm{HI}_{\mathrm{s}}+\mathrm{Dq}$ (see Appendix A), where $\mathrm{H}$ is the mean surface curvature and D is the deviatoric curvature. For cylindrical membranes of circular cross-section, the mean surface curvature $\mathrm{H}$ is equal to deviatoric curvature $\mathrm{D}$ and the curvature is constant throughout the surface and hence $\mathrm{D}=$ $\mathrm{H}=-(1 / 2 \mathrm{R})$, where $\mathrm{R}$ is the radius of the membrane. As the cross-section of the cylindrical membrane deviates from that of a circle to an ellipse or a super-ellipse, the curvature varies smoothly around the circumference and is a function of polar angle $\theta$. In this work, the crosssectional shape of the cylindrical membranes is represented by a family of super-ellipses. This family of curves is mathematically described by Lame' curves ${ }^{35,36,37}$ :

$$
\left|\frac{\mathrm{x}}{\mathrm{a}}\right|^{\eta}+\left|\frac{\mathrm{y}}{\mathrm{b}}\right|^{\eta}=1
$$

The shape parameter $\eta$ is any rational number that selects the shape of the membrane. The size parameters a and $\mathrm{b}$ correspond to the intersections of the curves with the $\mathrm{x}$ and $\mathrm{y}$ axes respectively and determine the size of the membrane. In this work, we consider only the subset of Lame' 
curves, where $\eta \geq 2$ and $a=b$. When $\eta=2$, eqn.(1) represents a circle and for $\eta>2$, the curve becomes a super-ellipse until it becomes a rectangle for $\eta \rightarrow \infty$. The curvature of a cylindrical membrane of super-elliptical cross-section at a given polar angle $\theta$ is given by: ${ }^{\mathbf{3 5}}$

$$
H=D=-\frac{(\eta-1)(x y)^{\eta-2}\left(x^{\eta}+y^{\eta}\right)}{2\left(x^{2 \eta-2}+y^{2 \eta-2}\right)^{\frac{3}{2}}}
$$

where $x=b /\left(1+\tan ^{\eta} \theta\right)^{1 / \eta}$ and $y=b \tan \theta /\left(1+\tan ^{\eta} \theta\right)^{1 / \eta}$. In this work, the variables $x, y$ and the parameter $\mathrm{b}$ are non-dimensionalized by scaling with the bare length scale of the model $\ell_{0}$, discussed in section 3.2.

\subsection{Fiber structure characterization}

The characterization of microfibril structure in the membrane is given by the $2 \times 2$ symmetric traceless quadrupolar tensor order parameter $\mathbf{Q}^{\mathbf{3 8 , 2 4} \text { : }}$

$\mathbf{Q}=\mathrm{S}\left(\mathbf{n n}-\frac{\mathbf{I}_{\mathrm{s}}}{2}\right)$

where $\mathrm{S}$ is the scalar order parameter that measures the microfibril alignment along the director $\mathbf{n}$, and $\mathbf{n} . \mathbf{m}=0$. The range of $\mathrm{S}$ is $-1 \leq \mathrm{S} \leq 1$, when $\mathrm{S}<0$ the microfibrils are aligned along $\mathbf{m}$, and when $S=0$ the microfibrils adopt an isotropic disordered state. In this paper we focus on nonnegative values of S. Since the curvature $\mathbf{b}$ has a trace $(\mathrm{H})$, the energy-coupling with nematic order involves a non-zero trace $2 \times 2$ symmetric tensor $\mathbf{S}$ with non-zero eigenvalues:

$$
\mathbf{S}=\mathbf{Q}+\frac{\mathbf{I}_{\mathrm{s}}}{2}=\left(\frac{\mathrm{S}+1}{2}\right) \mathbf{n n}+\left(\frac{1-\mathrm{S}}{2}\right) \mathbf{m m}
$$

\subsection{Membrane bending elasticity}

The elasticity of the fiber-laden membrane can be characterized by the effective elastic bending modulus that determines the rigidity of the fiber-laden membrane. The effective bending 
modulus $\mathrm{k}_{\mathrm{c}, \text { eff, }}$ which is necessarily positive, is defined as the free energy cost to deform a membrane from its intrinsic curvature to some other curvature and it is given by the second derivative of the total free energy $(\rho \hat{A})$ with respect to the mean surface curvature $\mathrm{H}$ :

$\mathrm{k}_{\mathrm{c}, \mathrm{eff}}=\frac{\mathrm{d}^{2}(\rho \hat{\mathrm{A}})}{\mathrm{dH}^{2}}$

The free energy of the fiber-laden membranes $(\rho \hat{A})$ in eqn.(6) is discussed in section 3.1.

\section{Model and predictions for curvature-induced fiber ordering}

In this section, the free energy of the fiber-laden membrane system is formulated and the resulting planar fiber orientation regimes are presented. The reader is referred to [24] for details. The model focuses on curvature-driven competing interactions, and does not account for spatial gradients.

\subsection{Free energies and fiber orientation regimes}

The total free energy per unit area $\rho \hat{\mathrm{A}}$ is posited to be:

$\rho \hat{\mathrm{A}}=\rho \hat{\mathrm{A}}_{\text {membrane }}(\omega, \mathrm{H}, \mathrm{K})+\rho \hat{\mathrm{A}}_{\text {fiber }}(\omega, \mathbf{Q})+\rho \hat{\mathrm{A}}_{\text {coupling }}(\omega, \mathbf{b} \cdot \mathbf{S})$

where $\rho$ is the density and $\omega$ is the fiber mass fraction; in this paper $\omega$ is fixed and will be omitted in what follows. The Helfrich free energy per unit area widely used to describe the elasticity of membranes reads ${ }^{39,40}$ :

$\rho \hat{\mathrm{A}}_{\text {membrane }}(\mathrm{H}, \mathrm{K})=\gamma_{\mathrm{o}}+2 \mathrm{k}_{\mathrm{c}}\left(\mathrm{H}-\mathrm{H}_{\mathrm{o}}\right)^{2}+\overline{\mathrm{k}}_{\mathrm{c}} \mathrm{K}$

where $\mathrm{k}_{\mathrm{c}}$ is the membrane bending elastic moduli, $\mathrm{H}_{\mathrm{o}}$ is the spontaneous curvature of the membrane, $\overline{\mathrm{k}}_{\mathrm{c}}$ is the torsion elastic moduli of the membrane; the effect of fiber concentration on $\mathrm{k}_{\mathrm{c}}$ 
and $\overline{\mathrm{k}}_{\mathrm{c}}$ is neglected in eqn.(7). The fiber contribution is given by the Landau-de Gennes expansion $^{38,41}$ :

$\rho \hat{\mathrm{A}}_{\text {fiber }}=\frac{\mathrm{a}_{1}}{2} \mathbf{Q}: \mathbf{Q}+\frac{\mathrm{a}_{4}}{4}(\mathbf{Q}: \mathbf{Q})^{2}$

where $a_{1}$ is a function of microfibril concentration and $a_{1}>0, a_{4}>0$ indicating that if nematic ordering arises at all, it is only through the fiber order-membrane curvature coupling; we note that this paper is restricted to dilute fiber suspensions for which $a_{1}>0$. The fiber order- membrane curvature coupling contribution up to second order terms in $\mathbf{S}$ is:

$\rho \hat{A}_{\text {coupling }}=\mathbf{b}:\left(\mathrm{a}_{2} \mathbf{S}+\mathrm{a}_{5} \mathbf{S} \cdot \mathbf{S}\right)+\frac{\mathrm{a}_{3}}{2}(\mathbf{b}: \mathbf{S})^{2}$

For $\mathrm{a}_{2}>0\left(\mathrm{a}_{2}<0\right)$ the first term $\mathbf{b}:\left(\mathrm{a}_{2} \mathbf{S}\right)$ promotes fiber alignment along the principal $\mathbf{e}_{1}\left(\mathbf{e}_{2}\right)$ direction; the same holds for $\mathrm{a}_{5}$, and hence they are denoted curvophilic. For $\mathrm{a}_{5}>0$, the second term $\mathbf{b}:\left(\mathrm{a}_{5} \mathbf{S} \cdot \mathbf{S}\right)$ promotes nematic ordering when the fibers are oriented along the principal axes (curvophilic). For $\mathrm{a}_{3}>0$, the third term $\mathrm{a}_{3}(\mathbf{b}: \mathbf{S})^{2} / 2$ promotes fiber orientation away from the principal axes (curvophobic). In biological fiber-laden membranes, curvophobic interactions may arise as an attempt to minimize the non-attachment penalty, the elastic energy cost associated with the rigid fibers not completely supported by the membrane when they are aligned in the direction of maximum curvature. On the other hand, curvophobic interactions may originate from any of the following mechanisms :(i) the fibers exhibiting preferential orientation over corrugated grooves on the membrane surface known as Berreman anchoring ${ }^{\mathbf{2}}$, (ii) intrinsic nanoscale coiling of biological fibers predominantly modeled by Helfrich elastic chiral filament model or Kirchhoff elastic theory of rods ${ }^{\mathbf{4 3}}$, (iii) membrane curvature induced by soft-mode instability of membranes caused due to the adsorbed fibers ${ }^{44}$. Other mechanisms applicable micron-ranged inclusions due to 
capillary process have been characterized by Stebe and co-workers ${ }^{\mathbf{4 5 , 4 6}}$. The balance between these curvophobic and curvophilic competing interactions is modified by the ambient curvature $\mathbf{b}$, since the power dependence is linear for $\left(a_{2}, a_{5}\right)$ and quadratic for $a_{3}$. The nature and meaning of the $a_{2}$ and $a_{3}$ terms has been previously discussed ${ }^{47}$, but the role of the curvophilic a in creating nematic order was not.

For a given geometry, the preferred fiber orientation $(\alpha)$ and fiber order $(S)$ is found by minimizing the Helmholtz free energy density $\rho \hat{A}$. By setting $\rho(\partial \hat{\mathrm{A}} / \partial \mathrm{S})=0$ and $\rho(\partial \hat{\mathrm{A}} / \partial \alpha)=0$ the following three equilibria states of fiber orientation arises: (a) orientation along major curvature or ring mode, $\alpha=0$; (b) along the minor curvature or line mode, $\alpha=\pi / 2$; and (c) oblique or helical mode, $\cos ^{2} \alpha=-\beta_{2} / 2 \beta_{4}$ as depicted in figure 1 .
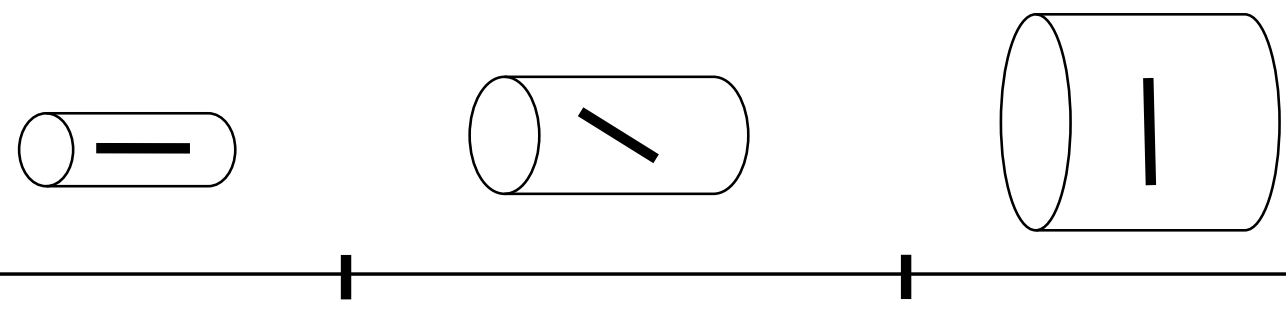

$\alpha=\pi / 2$

$\mathrm{R}_{2}$

$0<\alpha<\pi / 2$

$\mathrm{R}_{1}$

$\alpha=0$

Radius

Figure 1. Schematic of curvature-induced fiber orientation (thick line segment) on a cylinder of increasing radius R. At small (large) radius, the fibers align along the axial (azimuthal) direction. At intermediate curvatures, the oblique state minimizes the free energy (eqn.(13)). Adapted from [24].

The expression of the free energy in terms of $\mathrm{S}, \alpha, \mathrm{H}$ and $\mathrm{D}$, the minimization of the free energy, the conditions under which the three orientation regimes occur and their transitions radii $\left(\mathrm{R}_{1}, \mathrm{R}_{2}\right)$ are discussed in Appendix B.

\subsection{Fiber structure-curvature relations}


Next, the equations for fiber orientation regimes and their transitions (Appendix B) are solved for typical values of dimensionless energy coefficients $\left\{a_{i}\right\}$; we scaled the parameters with $a_{1}$ and use the same symbol for brevity. From the free energy we find the bare internal length scale $\ell_{\mathrm{o}}$ associated with the fiber orientation:

$$
\ell_{\mathrm{o}}=\frac{\mathrm{a}_{3}}{\mathrm{a}_{2}+\mathrm{a}_{5}}=\frac{\text { curvo }- \text { phobic energy }}{\text { curvo }- \text { philic energy }}
$$

Hence the characteristic radius of curvature or radius of the cylindrical membrane $(\mathrm{R})$ is scaled with the bare internal length scale $\ell_{o}$ such that the dimensionless radius $\mathrm{R}^{*}$ that controls the fiber orientation is the ratio of the external length scale to the internal length scale:

$$
\mathrm{R}^{*}=\frac{\text { external length }}{\text { internal length }}=\frac{\mathrm{R}}{\ell_{\mathrm{o}}}
$$

The selected dimensionless energy coefficients are $\left\{a_{i} ; i=1-5\right\}=[1,1,1,10,0.4]$. The large selected $\mathrm{a}_{4} / \mathrm{a}_{1}$ value reflects the fiber concentration condition close to the order/disorder transition, where $\mathrm{a}_{1}$ vanishes. The similar magnitudes in $\mathrm{a}_{1}, \mathrm{a}_{2}, \mathrm{a}_{3}$, and as reflect a condition of similar ordering and curvophobic and curvophilic effects for dilute fiber concentrations below the nematic transition.

Figure 2 depicts the fiber orientation modes in terms of cosine of equilibrium fiber orientation angle with respect to azimuthal coordinate $(\cos \alpha)$ and equilibrium fiber order in terms of scalar order parameter (S). At low membrane radius, for $0<R^{*}<0.1847$, the curvophilic free energy is negligible and the curvophobic free energy is minimized, thus the fibers are aligned in the axial direction resulting in the line mode. At intermediate membrane radius, for $0.1847<\mathrm{R}^{*}<0.3867$, the competition between the fiber interactions and curvophilic free energy aligns the fibers at an angle between $0-90^{\circ}$, resulting in the helical mode. At high membrane 
radius, for $\mathrm{R}^{*}>0.3867$, the curvophobic free energy is negligible and the curvophilic free energy is minimized, thus the fibers are aligned in the azimuthal direction resulting in the ring mode. At low membrane radius, the fibers are more uniformly aligned in the axial direction. As the membrane radius increases, the fiber order $\mathrm{S}$ decreases until a local minimum (cusp) is reached, due to the cancellation of free energy contributions from curvophobic and curvophilic interactions. At the onset of the ring mode, the fiber order $\mathrm{S}$ starts increasing until a local maximum is reached promoted by curvophilic interactions. At high membrane radii, the fiber order vanishes asymptotically as the membrane curvature vanishes resulting in random fiber orientation.

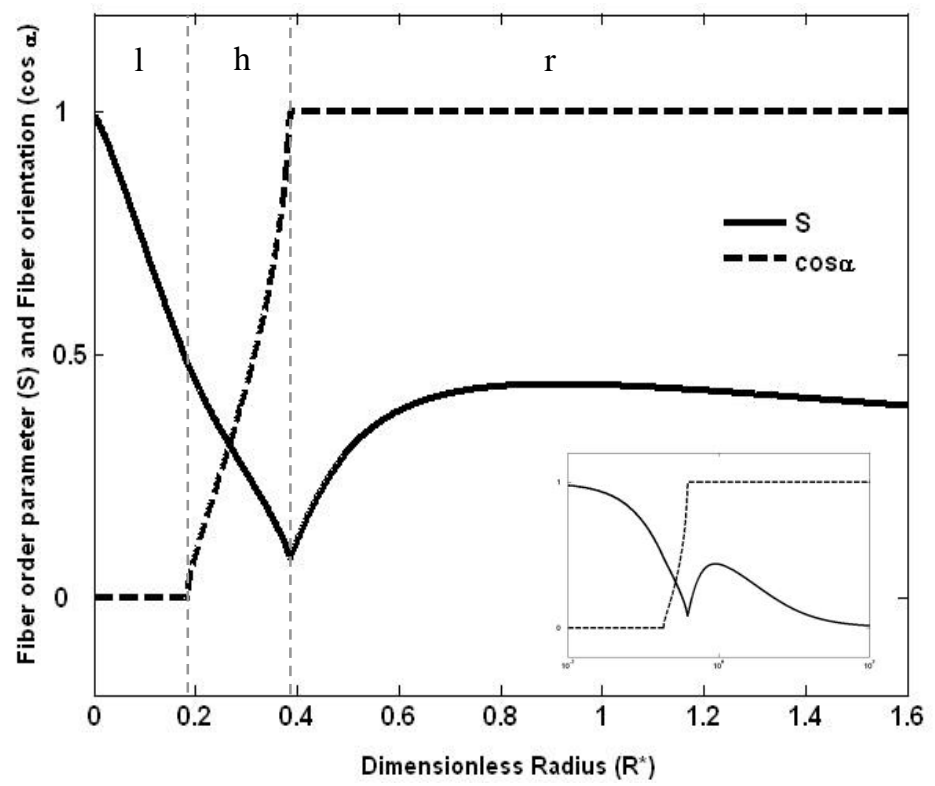

Figure 2. Fiber structure plot for cylindrical membranes of circular cross-section. The three possible modes are: 1 - line mode, $\mathrm{h}$ - helical mode, $\mathrm{r}$ - ring mode. The order parameter displays a typical $\mathrm{V}$ shape and the orientation a step-like response as the radius increases. The inset semi-log plot shows fiber order vanishing asymptotically as $\mathrm{R}^{*} \rightarrow \infty$.

\subsection{Model validation for fiber orientation regimes}

Although the present model does not take into account temporal variation of cellulose microfibril concentration during different stages of cell wall deposition, the predicted fiber 
orientation (see Figure 2) is in qualitative agreement with the experimental observations for the orientation of cellulose microfibrils in cell walls of trachieds of conifers based on field emission scanning microscopy ${ }^{48}$. The progressive changes in the orientation of cellulose microfibrils in primary and secondary layers of these plant cell walls are shown in figure 3(a). A schematic of cell wall deposition process and corresponding changes in membrane shape are depicted in figure 3(b). The initial layers of primary cell wall are deposited at the onset of cell growth when the membrane radius is small, resulting in line mode (Figure 3(a)-1, 3(b)-1). The final layers of primary cell wall are deposited towards the end of cell growth, when the membrane radius is large as the cell reaches its maximum size (Figure 3(b)-2). Consequently, the layers deposited in this phase exhibit ring mode (Figure 3(a)-2). This result is in agreement with experimental observations showing the orientation of microfibrils changing from line to ring mode in different plant species ${ }^{48-50}$. When the cell growth ceases the secondary cell wall layers are deposited from the inner side and the cell starts shrinking to become a lumen. As the radius of the membrane decreases due to the cell wall deposition, the fiber orientation changes to helical mode in the $S_{1}$ layer and the fiber angle with respect to azimuthal direction progressively changes from $0-90^{\circ 46}$. The $S_{2}$ layers deposited at further smaller membrane diameter exhibits further steeper fiber orientation closer to the line mode and show high fiber order $^{45}$ (Figure 3(a)-3, 3(b)-3). The discrepancy between the experimentally observed helical or crossed helical modes and the model based prediction of line mode in $\mathrm{S}_{3}$ layer might be attributed to lignification of the $S_{3}$ layers before completion of cellulose microfibril alignment, as $S_{3}$ is the innermost layer deposited towards the end of the cell wall deposition process. This reasoning is further supported by wide variations in observed microfibril orientation in innermost layers of $S_{3}$ among different tree species ${ }^{48}$. As the fiber structure of $S_{3}$ layer is determined by the duration of deposition and the rate of change in orientation of microfibrils prior 
to lignification, modelling the temporal dynamics of fiber orientation might be able to resolve this discrepancy.

a

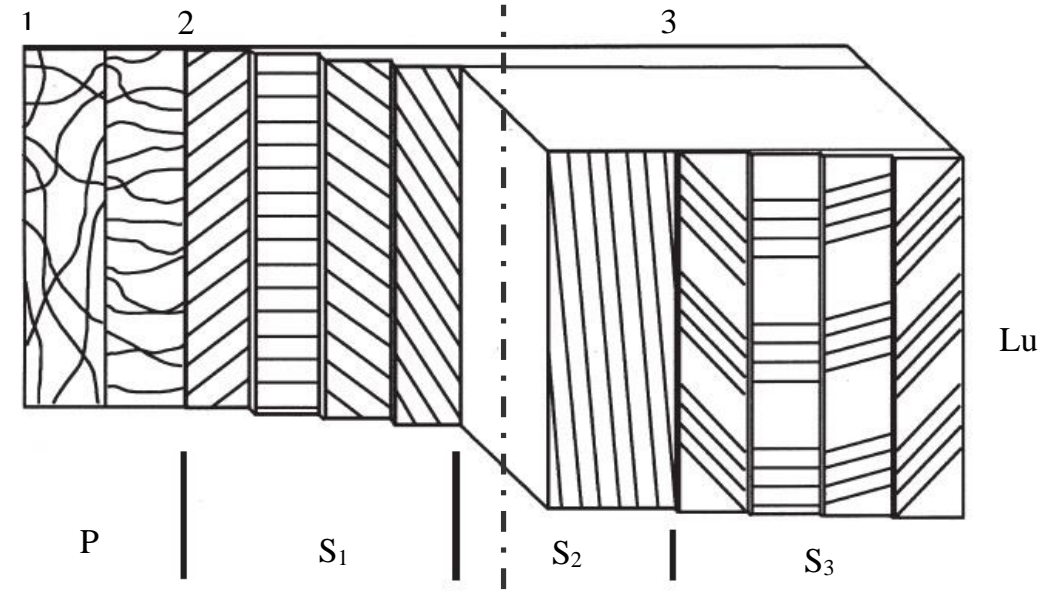

b

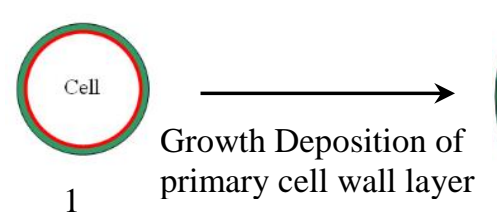

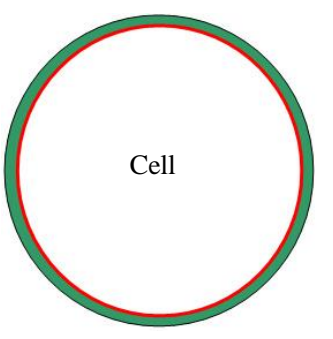

2

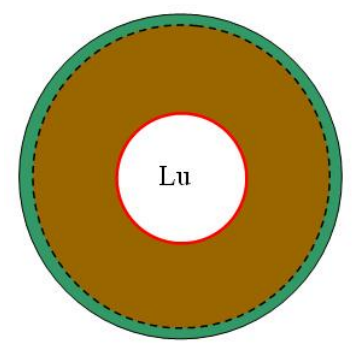

3

Figure 3. (a) A model for the orientation of cellulose microfibrils viewed from the lumen side in the cell walls of tracheids of conifers. $\mathrm{P}$ - Primary cell wall, $\mathrm{S}_{1}, \mathrm{~S}_{2}, \mathrm{~S}_{3}$ - Secondary cell wall layers 1, 2, 3, Lu - Lumen. Adapted from [48]. (b) A schematic of cell wall deposition process and corresponding changes in membrane shape. Lu - Lumen. The changes in curvature correspond to different fiber orientation and order, as per Figure 2.

\subsection{Effective bending modulus of cylindrical fiber-laden membrane of circular cross-section}

To gain insight into the role of fiber structure on the elasticity of fiber-laden membranes, the effect fiber orientation and fiber order on the effective bending modulus of the fiber-laden membrane cross-section is investigated. In this $2 \mathrm{D}$ analysis, bending denotes changes in curvature in the cross-sectional plane and not along the membrane axis. The effective bending modulus of an arbitrarily curved fiber-laden membranes $\mathrm{k}_{\mathrm{c}, \text { eff }}$ has contributions due to membrane bending 
elasticity, $\mathrm{k}_{\mathrm{c}, \text { membrane, }}$ and membrane curvature induced nematic ordering of fibers, $\mathrm{k}_{\mathrm{c}, \text { fiber. }}$. For cylindrical membranes in which the fiber order is induced through coupling between fiber order and membrane curvature, $H=D=-(1 / 2 R)$, and fiber orientation, $\alpha$ and order, $S$ are functions of the dimensionless radius of curvature of the membrane $\mathrm{R}^{*}$ :

$\mathrm{k}_{\mathrm{c}, \text { fff }}\left(\mathrm{R}^{*}\right)=\mathrm{k}_{\mathrm{c}, \text { fiber }}\left(\mathrm{R}^{*}, \alpha\left(\mathrm{R}^{*}\right), \mathrm{S}\left(\mathrm{R}^{*}\right)\right)+\mathrm{k}_{\mathrm{c}, \text { membrane }}\left(\mathrm{R}^{*}\right)$

The expressions for $\mathrm{k}_{\mathrm{c}, \text { eff }}$ in the three fiber orientation modes are derived in Appendix C.

The effective bending modulus of the fiber-laden membrane system $\mathrm{k}_{c, \text { eff }}$ is plotted as a function of the dimensionless radius of the membrane $\mathrm{R}^{*}$ in figure 4 . The effective bending modulus of the fiber-laden membrane system results from competing interactions between curvophobic and curvophilic interactions, and bare membrane bending modulus. It is evident from figures 2 and 6 that the effective bending modulus is sensitive to fiber order $\mathrm{S}$ when the fibers are oriented in ring mode and is independent of fiber order when the fibers are oriented in line or helical mode. Depending on the role of fibers on the bending elasticity of the fiber-laden membranes, three distinct regions have been identified, as follows:

(i) Pure membrane regime, $0<\mathrm{R}^{*}<0.3867$, region (i) in Figure 4: In this region, the effective bending modulus $\mathrm{k}_{\mathrm{c}, \text { eff }}$ is almost equal to the bending modulus contribution from the membrane

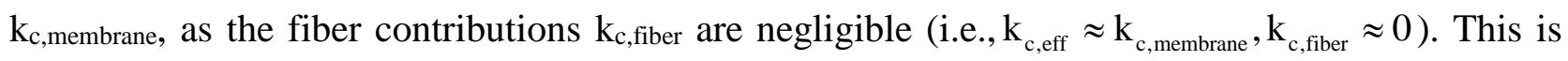
due to a lack of resistance to change in membrane curvature when the fibers are oriented in the line or helical mode. The addition of fibers neither strengthens the membrane nor destabilizes the membrane shape. The bending elasticity of the fiber-laden membranes is not influenced by the presence of fibers.

(ii) Membrane hardening regime, $0.3867<\mathrm{R}^{*}<4.175$, region (ii) in Figure 4 ; for $\mathrm{R}^{*}>0.3867$, the fibers oriented in ring mode offer resistance to change in membrane curvature proportional to the 
magnitude of fiber order parameter S. The theoretical maximum effective bending modulus, which is the value obtained when the fibers are oriented in ring mode and the fiber order parameter $\mathrm{S}$ equals 1 , is never realized as the maximum observed fiber order $\left(S_{\max }\right)$ in ring mode for the typical values of energy coefficient used in this work is 0.439 at $R^{*}=0.913$. The corresponding maximum effective bending modulus for the fiber-laden membrane system under investigation is 6.071. As $\mathrm{R}^{*}$ is increased from 0.3867 to $0.913, \mathrm{dS} / \mathrm{dR}^{*}>0$ and $\mathrm{S} \rightarrow \mathrm{S}_{\max }$ and hence the fibers oriented in the ring mode become more ordered resulting in gradual strengthening of the membrane by the fibers, against deformation through change in curvature. For $\mathrm{R}^{*}>0.913$, gradual randomization of the fibers begins $\left(\mathrm{dS} / \mathrm{dR}^{*}<0\right)$ as the membrane curvature diminishes asymptotically. This results in the steady decrease in effective bending modulus at values of $R^{*}>0.913$. For $0.913<R^{*}<4.175$, the curvophobic interactions dominate over curvophilic interactions, resulting in finite positive fiber bending modulus contribution (i.e., $\mathrm{k}_{\mathrm{c}, \text { fiber }}>0, \mathrm{k}_{\mathrm{c}, \text { eff }}>\mathrm{k}_{\mathrm{c}, \text { membrane }}$ ) and the fibers continue to strengthen the membrane against deformation through change in curvature.

(iii) Membrane elastic softening regime, $R^{*}>4.175$, region (iii) in Figure 4: At $R^{*}=4.175$, the fiber contribution to the effective bending modulus vanishes as the curvophobic and curvophilic interactions cancel each other and hence the effective bending modulus of the fiber-laden membranes is that of a pure membrane (i.e., $\mathrm{k}_{\mathrm{c}, \text { eff }}=\mathrm{k}_{\mathrm{c} \text {, membrane }}$ ). At values of $\mathrm{R}^{*}>4.175$, the curvophilic interactions dominate the curvophobic interactions, resulting in finite negative fiber bending modulus contributions (i.e., $\mathrm{k}_{\mathrm{c}, \text { fiber }}<0$ ) and fibers tend to destabilize the membrane shape. This destabilizing effect induced by the fibers is compensated by the membrane bending modulus contribution, thus maintaining the effective bending modulus of the fiber-laden system positive (i.e. $0<\mathrm{k}_{\mathrm{c} \text {,eff }}<\mathrm{k}_{\mathrm{c} \text {,membrane }}$ ). This competing interaction between the fiber and membrane 
contributions at values of $\mathrm{R}^{*}>4.175$, helps sustaining the shape stability of the fiber-laden membrane system, but results in membranes of low effective bending modulus that are more susceptible to deformation. This elastic softening observed at values of $R^{*}>4.175$ is attributed to the bilinear coupling between the order parameter and curvature, and is commonly observed in systems with one order parameter (such as Q) coupled to strain (such as b) or systems with multiple order parameters ${ }^{51}$. For the selected parametric data, as the fiber order asymptotically vanishes ast $\mathrm{R}^{*} \rightarrow \infty$, the effective bending modulus of the fiber-laden membrane system stabilizes at 1.084 .

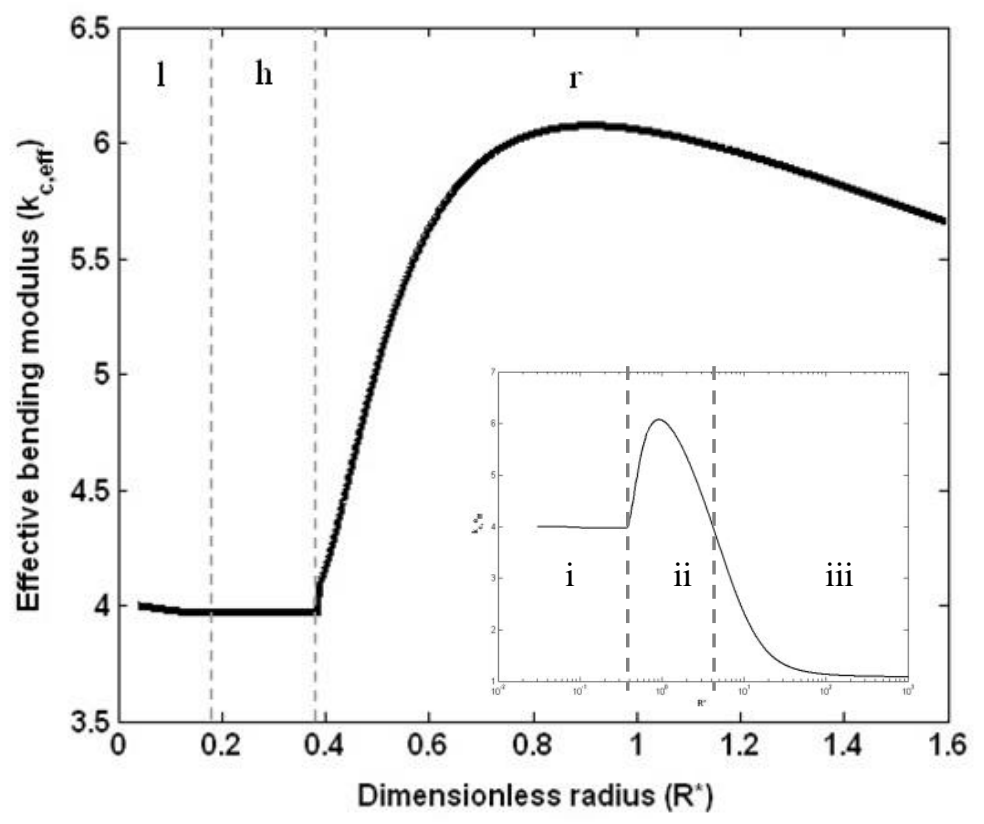

Figure 4. Effective bending modulus plot for cylindrical membranes of circular cross-section. 1 - line mode, $\mathrm{h}-$ helical mode, $r$ - ring mode. The inset semi-log plot shows region (i) pure membrane regime $\mathrm{k}_{\mathrm{c}, \mathrm{eff}}=\mathrm{k}_{\mathrm{c}, \mathrm{membrane}}$, (ii)

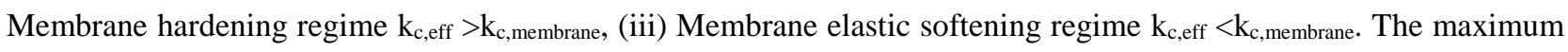
stiffening occurs when the membrane radius $\mathrm{R}$ is nearly equal to the internal length scale $\ell_{\mathrm{o}}$.

Figure 5 shows the bending stiffness phase plot in terms of radius of curvature of a circular membrane $(\mathrm{R})$ as a function of the bare internal length scale of the material $\left(\ell_{\mathrm{o}}\right)$, identifying the 
three membrane elasticity regimes (i-iii) discussed in Figure 4. For any fiber-filled circular membrane the bare internal length scale $\left(\ell_{\mathrm{o}}\right.$, eqn.(10)) is fixed and the membrane displays growthinduced bending variability as a result of changes in membrane radius (R). The maximum stiffening of the membranes occurs on the dotted line in the plot, when the membrane radius $\mathrm{R}$ is nearly equal to the internal length scale $\ell_{\mathrm{o}}$. For given material, increasing $\mathrm{R}$ first generates no fiber effect, subsequently the fibers stiffen the membrane, and eventually the fibers soften the circular membrane.

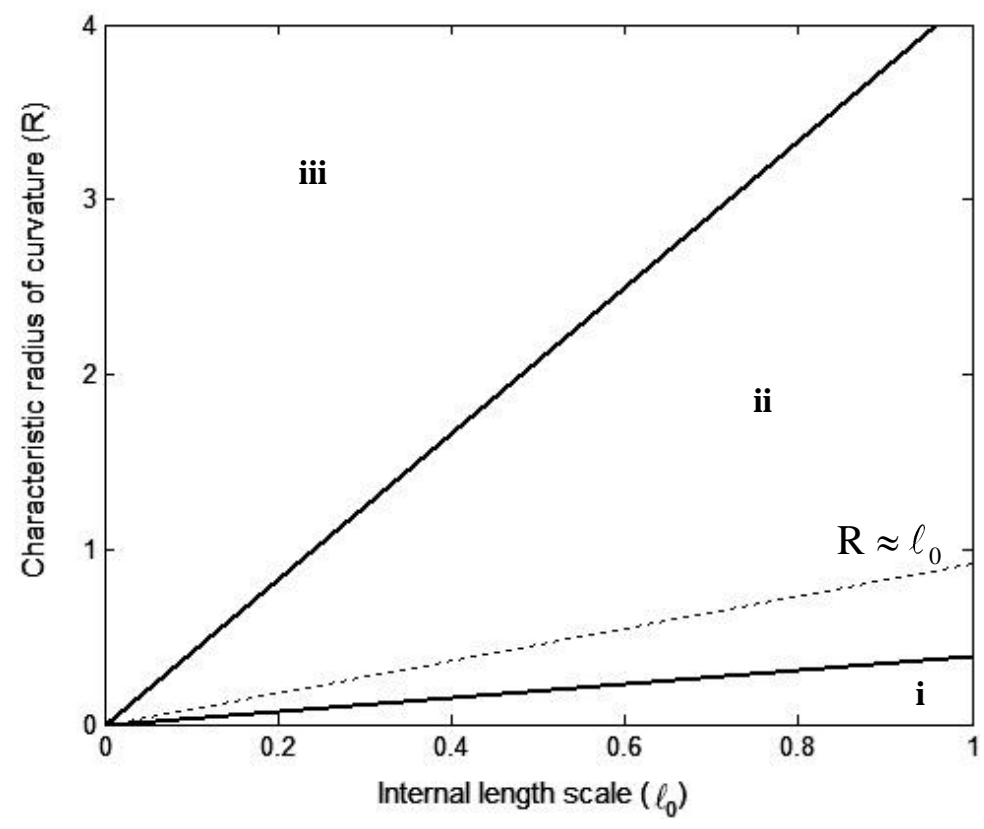

Figure 5.Bending stiffness phase plot in terms of circular membrane radius (arbitrary units) as a function of the internal length scale of the material (eqn.(10)). Region (i) pure membrane regime, (ii) membrane hardening regime, and (iii) membrane softening regime. The dotted line represented the line of maximum membrane stiffness.

In partial summary, fibers have the strongest effect in increasing bending stiffness of the cross section when they are strongly mutually aligned (high S) and with an average orientation in the azimuthal direction (region ii in figure 4) and this occurs when the membrane radius $\mathrm{R}$ is essentially equal to the internal length scale of material $\ell_{0}$. When the fibers are disordered they promote elastic softening (region iii in figure 4), and when they are axially oriented they have 
essentially no effect. During cell growth, the variation of bending stiffness can serve as a shape lock-in mechanism (hardening regime) or conversely allow shape change through the softening effect.

\section{Curvature-induced fiber ordering in cylindrical membranes of super-elliptical cross- section}

In this section, the fiber structure $(\mathbf{Q})$ in cylindrical fiber-laden membranes of superelliptical cross-section is analyzed based on the fiber orientations and their transitions predicted in section 3.2. The key issue is to characterize the effect of membrane curvature gradients $\left(\nabla_{s} \mathbf{b}\right)$ inherent in super-elliptical shapes on the fiber order parameter gradients $\left(\nabla_{s} \mathbf{Q}\right)$, using the circular shape as reference.

\subsection{Effect of shape parameter on fiber structure}

As stated in section 2.1, the membrane cross-sectional shapes observed in plant cell walls has been represented by Lame' curves. The fiber structure plots for $\eta \geq 2$ and $b=1$ for the typical values of energy coefficient used in section 3.2 are presented in figure 6 . The intercept of the curves on $\mathrm{x}$ and $\mathrm{y}$ axes are fixed by setting the size parameter $\mathrm{b}$ equal to 1 while varying the shape parameter $(\eta)$, resulting in a set of curves shown in figure 6(a). The curves being symmetric, calculating the fiber structure for a quadrant of the membrane is sufficient.

At $\eta=2$, the general equation for the Lame' curves represents a circle of constant curvature around the membrane's circumference. Planar self assembly of fibers driven by membrane curvature in these membranes results in microfibrils aligning in a specific direction at a uniform fiber order throughout the membrane. The resulting fiber structure has unimodal fiber orientation 
and uniform fiber order. The fiber orientation and order are dictated by the fiber structure plot in figure 2.

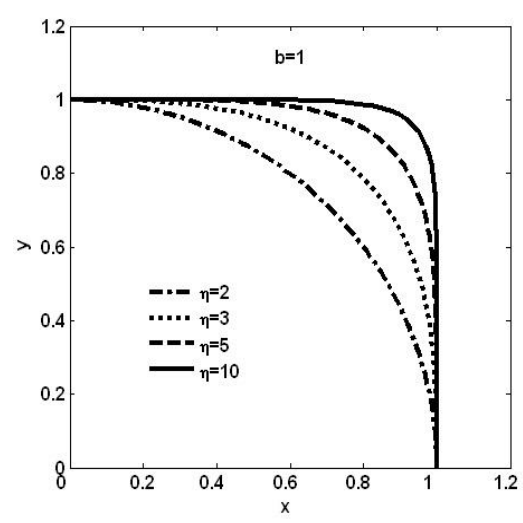

b

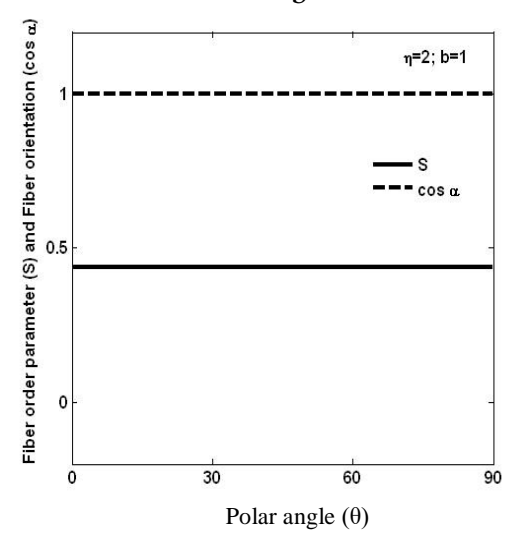

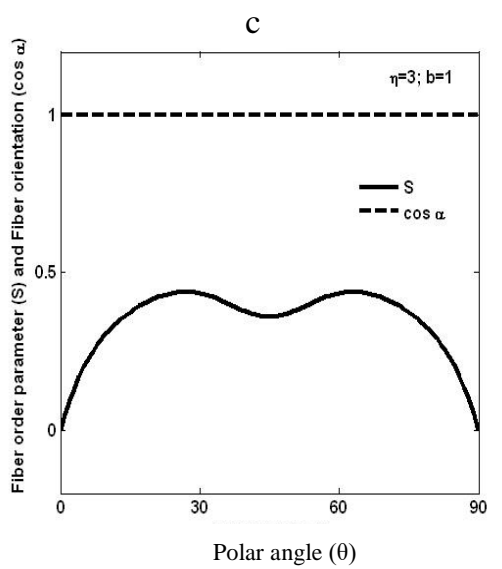
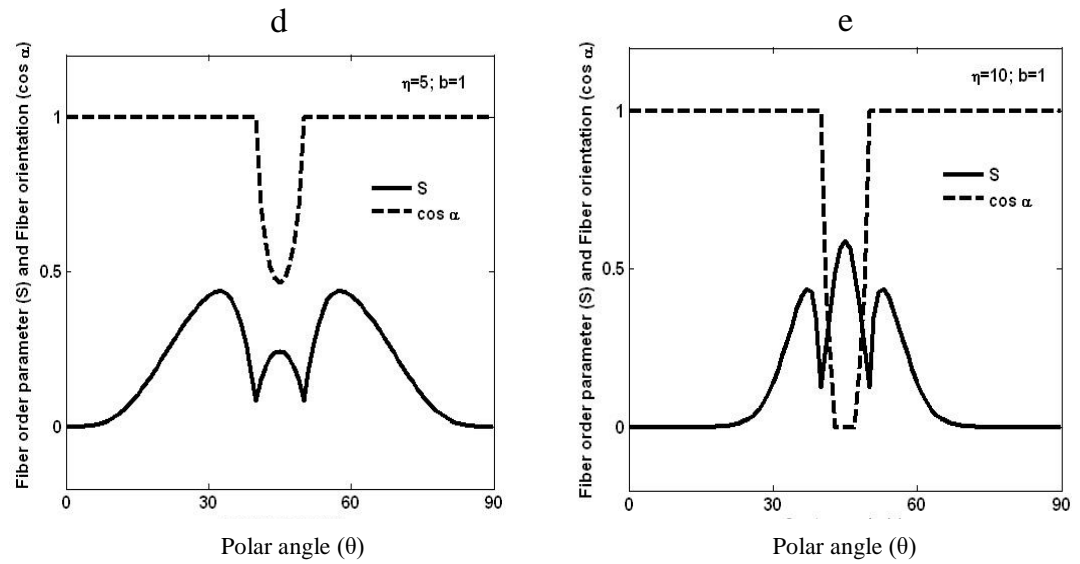

Figure 6. Fiber structure plot for cylindrical membranes of super-elliptical cross-section. (a) Lame' curves for $\eta=2,3,5,10$ and $b=1$, (b) Fiber structure plot for $\eta=2$ and $b=1$, (c) Fiber structure plot for $\eta=3$ and $b=1$, (d) Fiber structure plot for $\eta=5$ and $b=1$, (e) Fiber structure plot for $\eta=10$ and $b=1$. Increasing and localizing curvature produces sharp and localized fiber structure changes.

For $\eta=2$ and $b=1$, the fibers are aligned in ring mode (Figure $6(b)$ ). For $\eta>2$, the crosssectional shape transforms the circle into a family of super-ellipses resulting in curves with flat sides of large characteristic radius of curvature and corners of small characteristic radius of curvature. As $\eta$ is progressively increased, the variation in radius of curvature between the sides and the corners becomes increasingly pronounced and results in heterogeneous fiber orientation. 
For $\eta=3$ and $b=1$, the gradient in radius of curvature is not large enough to induce different fiber orientations. As the result, the fibers are aligned in ring mode throughout the membrane but the fiber order varies smoothly around the membrane circumference as a function of membrane curvature (Figure 6(c)). The resulting fiber structure has unimodal fiber orientation and nonuniform fiber order. For $\eta=5$ and $b=1$, the gradient in radius of curvature induces a ring mode in the sides of larger radius of curvature and a helical mode in the corners of smaller radius of curvature (Figure 6(d)). The resulting fiber structure has bimodal fiber orientation and non-uniform fiber order. For $\eta=10$ and $b=1$, the gradient in the radius of curvature induces ring mode in the sides of larger radius of curvature, line mode in the corners of smaller radius of curvature and helical mode in the regions of intermediate radius of curvature (Figure 6(e)). The resulting fiber structure has trimodal fiber orientation and non-uniform fiber order.

These numerical predictions are consistent with abrupt change in fiber angle in $S_{2}$ and $S_{3}$ at the corners reported in plant cell walls of juniper tracheid. The fiber orientation angle in the corners measured with respect to azimuthal coordinate $(\alpha)$ is 15 to $25^{\circ}$ greater than in the sides ${ }^{\mathbf{1 4}}$.

\subsection{Effect of size parameter on fiber structure}

For a given cross-sectional shape of a plant cell, the size of the membrane increases during growth and decreases during secondary cell wall deposition. The effect of varying the membrane size on the fiber structure, while fixing the membrane shape is investigated in this section. The shape of the membrane is fixed by setting the shape parameter $\eta$ equal to 5 while varying the intercept of the curves on $x$ and $y$ axes (b), resulting in a set of curves shown in figure 7(a). As b is progressively decreased, the radius of curvature of the corners decreases drastically in comparison to that of the sides resulting in a greater curvature gradient between the corners and the sides. This 
difference in curvature might result in different fiber orientation modes in the sides than the corners.
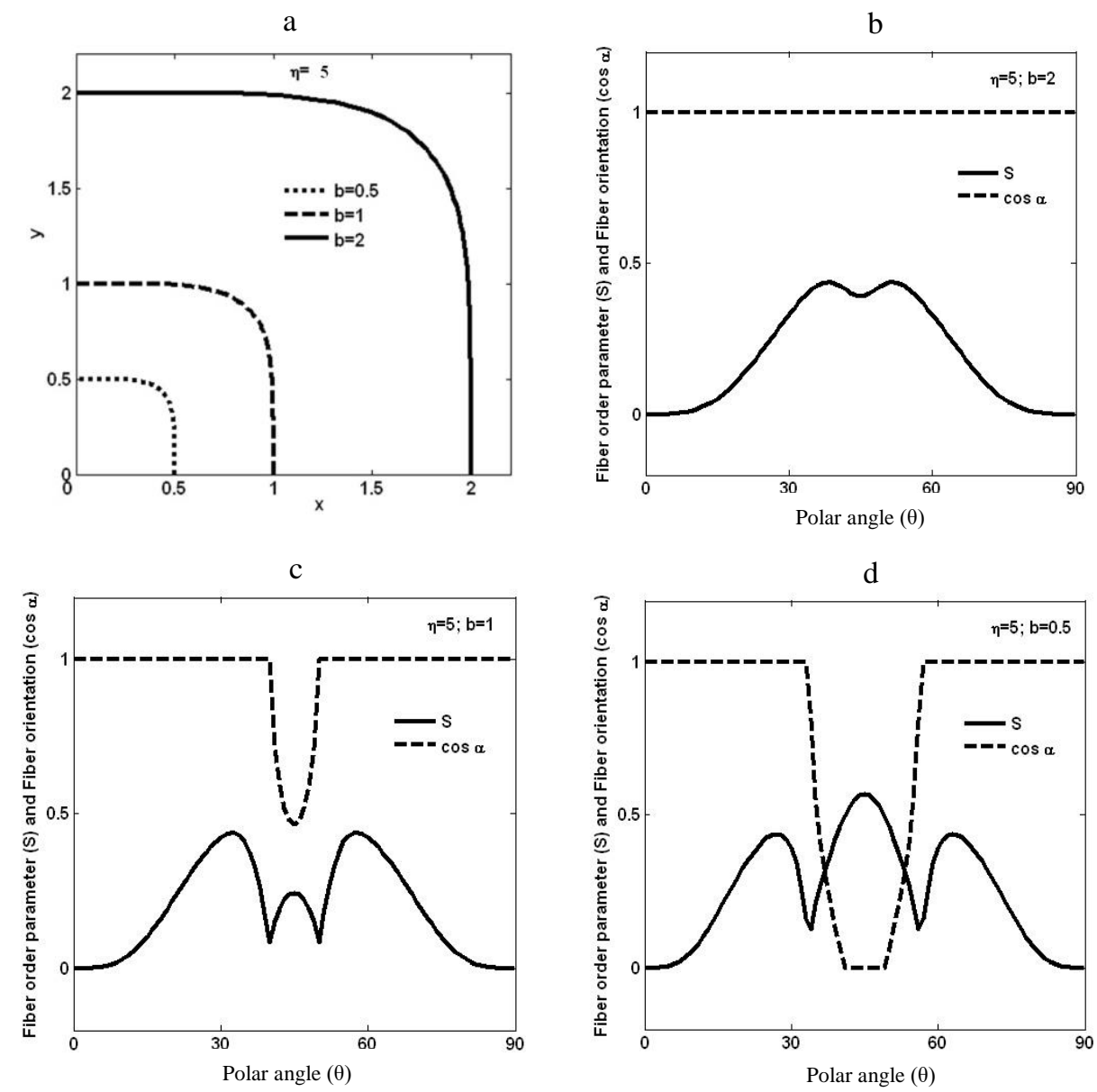

Figure 7. Fiber structure plot for cylindrical membranes of super-elliptical cross-section. (a) Lame' curves for $b=2,1,0.5$ and $\eta=5$, (b) Fiber structure plot for $\eta=5$ and $b=2$, (c) Fiber structure plot for $\eta=5$ and $b=1$, (d) Fiber structure plot for $\eta=5$ and $b=0.5$.

For $\eta=5$ and $b=2$, the gradient in radius of curvature is not large enough to induce different fiber orientations. As a result, the fibers are aligned in ring mode throughout the membrane but the fiber order varies smoothly around the membrane circumference as a function of membrane curvature (Figure 7(b)). The resulting fiber structure has unimodal fiber orientation and nonuniform fiber order. For $\eta=5$ and $b=1$, the gradient in radius of curvature induces ring mode in the sides of larger radius of curvature and helical mode in the corners of smaller radius of curvature 
(Figure 7(c)). The resulting fiber structure has bimodal fiber orientation and non-uniform fiber order. For $\eta=5$ and $b=0.5$, the gradient in radius of curvature induces ring mode in the sides of larger radius of curvature, line mode in the corners of smaller radius of curvature and helical mode in the regions of intermediate radius of curvature between the sides and the corners (Figure 7(d)). The resulting fiber structure has trimodal fiber orientation and non-uniform fiber order.

An experimental investigation supported by analytic and computational predictions on the mechanical advantages of having graded fiber orientation in man-made fiber-reinforced composite materials has concluded that under tensile stresses induced by indentation, a composite material with linearly graded fiber orientation exhibits greater resistance to microcracking ${ }^{\mathbf{5 2}}$. In addition to fiber orientation, the fiber orientation distribution is also known to influence all the elastic moduli of composite materials ${ }^{\mathbf{5 3}}$ and hence the uniformity of fiber orientation distribution is a crucial factor that determines the elastic homogeneity and hence the modulus of the composite material. In biological fiber-laden membranes, shape fluctuations arising due to elastic inhomogeneities are known to induce local change in bending rigidity and spontaneous membrane curvature ${ }^{54}$ and play a crucial role in morphogenesis in biological systems ${ }^{\mathbf{5 5}}$. Hence, an understanding of deformation modes in cylindrical membranes of super-elliptical cross-section due to spatial variation of membrane bending modulus is crucial.

To gain insight into the effect of membrane geometry on these factors influencing the mechanical efficiency of fiber-reinforced composites, a fiber structure phase map is developed in section 4.3. The spatial distribution of the effective bending modulus in cylindrical fiber-laden membranes of super-elliptical cross-section and their characteristic deformation modes are presented in section 4.4 . 


\subsection{Fiber structure phase map for cylindrical membranes of circular and super-elliptical cross-section}

As illustrated in sections 4.1 and 4.2 , in plant cell walls, multimodal fiber orientations resulting in graded fiber orientation may arise both in radial and azimuthal directions due to curvature gradients between the sides and the corners induced by the shape of plant cells and change in its size during cell growth and secondary cell wall deposition. To characterize the number of coexisting modes and the degree of heterogeneity we collapse all the computational results into master plots that summarize the impact of size and shape on these important indices.

Figure 8 shows a phase diagram in terms of the shape parameter as a function of the size parameters indicating the number of coexisting modes on a given cross-section. The unimodal (a) domain might correspond to fibers oriented in either ring, helical or line mode, the bimodal (b) domain corresponds to fiber structures with coexisting ring and helical modes or helical and line modes, and the trimodal (c) domain corresponds to fiber structures containing the three modes.

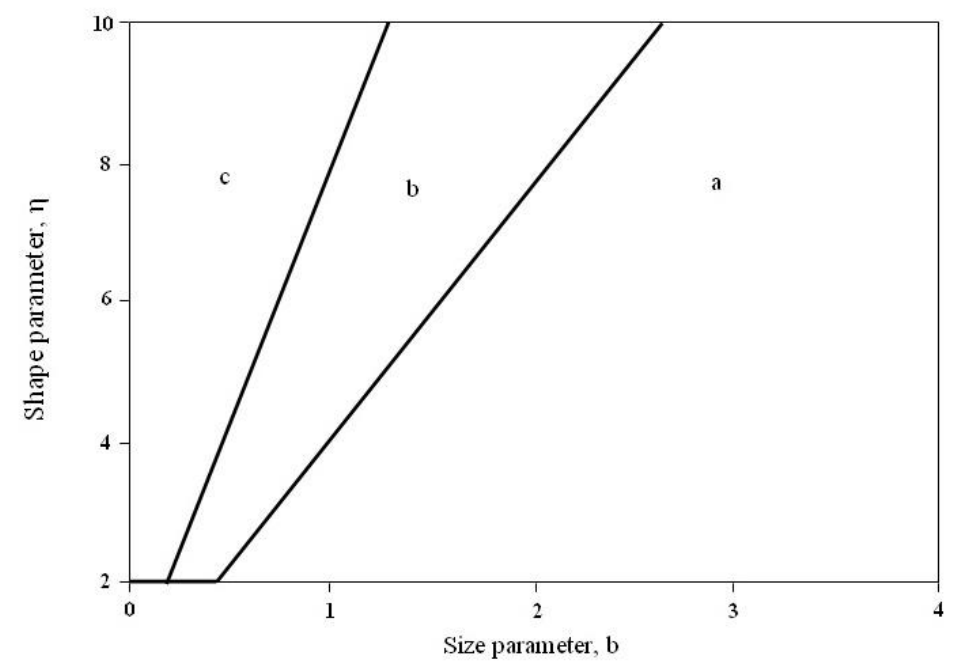

Figure 8. Phase map for fiber orientation modes in cylindrical plant cell walls of circular and super-elliptical crosssection. a - Unimodal, b- bimodal, c- trimodal fiber orientation modes

Figure 8 demonstrates that an increase in mode coexistence is attained through either membrane size reduction or by deviation from membrane constant curvature. 
The uniformity in fiber orientation distribution is characterized using the following nonuniformity index $\mathrm{U}$ :

$$
\mathrm{U}=\int_{0}^{\pi / 2}\left|\frac{\mathrm{d} S}{\mathrm{~d} \theta}\right| \mathrm{d} \theta .
$$

When $\eta=2$, the fiber order is uniform and the non-uniformity index is $U=0$. In the range of $2.25<\eta<10,0.05<\mathrm{b}<4$ explored in this work, the maximum value of $\mathrm{U}$ is $3.77 \times 10^{-2}$, which corresponds to $\eta=9$ and $b=0.05$. We introduce the following index classification: (i) high uniformity: $0<\mathrm{U}<1 \times 10^{-2}$, (ii) intermediate uniformity: $1 \times 10^{-2}<\mathrm{U}<2 \times 10^{-2}$, and (iii) low uniformity $2 \times 10^{-2}<\mathrm{U}<3.77 \times 10^{-2}$. Figure 9 shows the uniformity index phase diagram in terms of shape $\eta$ and size $b$ parameters.

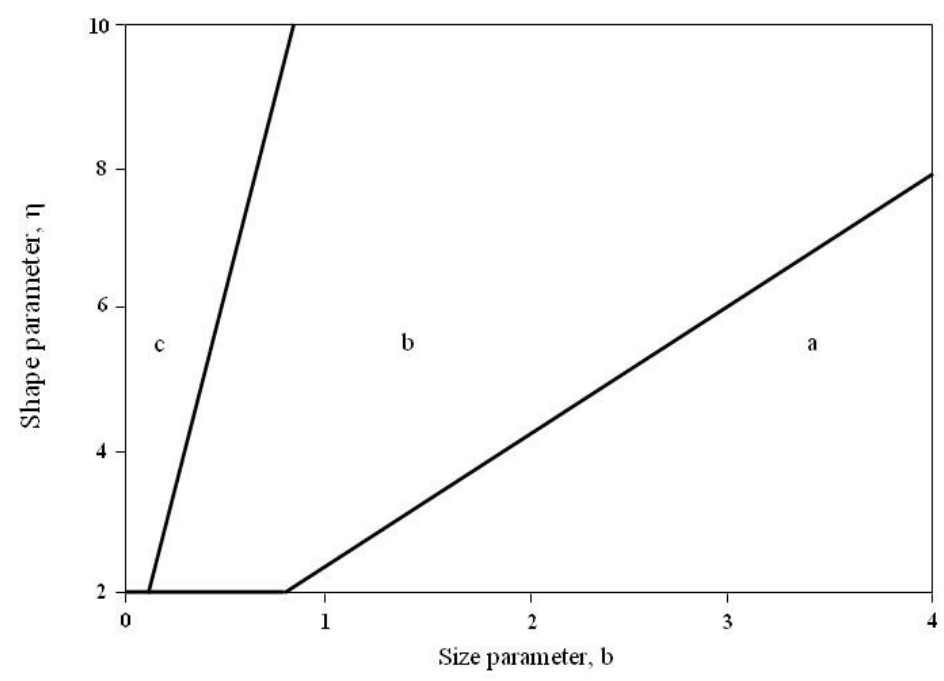

Figure 9. Phase map for uniformity in fiber orientation distribution in cylindrical plant cell walls of circular and superelliptical cross-section. a - high uniformity, b- intermediate uniformity, c- low uniformity

Figure 9 shows that low uniformity is promoted by size reduction and variable curvature. Under sufficiently small size, even modest deviations from circular shape create large heterogeneity.

As mentioned above a composite material with linearly graded fiber orientation exhibits greater resistance to microcracking ${ }^{52}$, while the uniformity of fiber orientation distribution is known to influence all the modulus of the composite materials ${ }^{\mathbf{5 3}}$. Based on these facts, we may 
expect that region "c" in Figure 8 have better resistance of microcracking. In figure 9, we may expect region "a" to have higher modulus due to homogeneous membrane elastic properties. Transferring the rules of man-made composites to our biological system ${ }^{\mathbf{5 2 5 3}}$, the phase maps indicate trade-off between microcracking resistance and strength in plant cell walls of superelliptical cross-section. Obviously, further experimental characterization and simulation is needed to put these claims on firm ground but they highlight potential mechanical optimization mechanisms in plants.

\subsection{Effective bending modulus of fiber-laden membranes of super-elliptical cross-section}

It is evident from figure 4 that the effective bending modulus $k_{c, \text { eff }}\left(R^{*}\right)$ is a function of the dimensionless radius of curvature $\mathrm{R}^{*}$. As the membrane curvature varies smoothly across the circumference of super-elliptical membranes, the effective bending modulus is spatially inhomogeneous. As mentioned above under stress, the softened section with low bending elastic modulus deforms while the stiffened sections with high bending elastic modulus remains intact resulting in membrane shape distortions. In this section, the effective bending modulus of cylindrical fiber-laden membranes of super-elliptical cross-section is computed for typical values of shape and size parameter, and their potential deformation modes under further expansion are analyzed.

Figure 10 shows the effective bending modulus $k_{c, \text { eff }}\left(R^{*}\right)$ as a function of the polar angle $\theta$ for three distinct super-elliptical membranes (a) corresponding to: (i) $\eta=2$ and $b=0.5$ (b), (ii) $\eta=4$ and $b=0.75$ (c), and (iii) $\eta=6$ and $b=3.5$ (d). The polar angle is measured by $\theta$ from the $x$-axis. Below we make reference to the corners which are at $\theta=+45^{\circ}$ and to the midpoint of the sides which are at $\theta=0,+90^{\circ}$. 

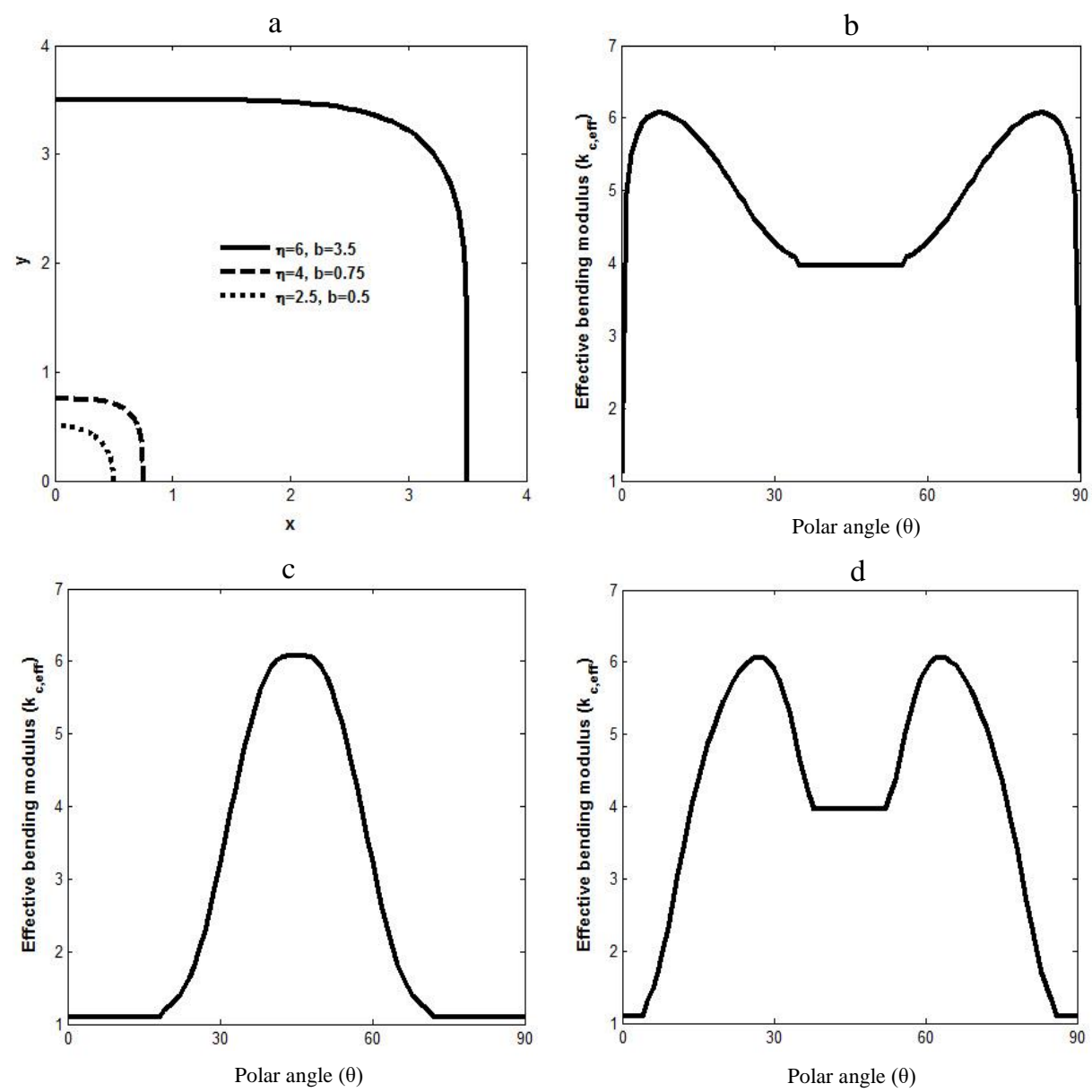

Figure 10. Effective bending modulus plot for cylindrical membranes of super-elliptical cross-section. (a) Lame' curves for, (i) $\eta=2.5$ and $b=0.5$, (ii) $\eta=6$ and $b=3.5$, and (iii) $\eta=4$ and $b=0.75$ (b) Effective bending modulus plot for $\eta=2.5$ and $b=0.5$, (c) Effective bending modulus plot for $\eta=6$ and $b=3.5$, (d) Effective bending modulus plot for $\eta=4$ and $\mathrm{b}=0.75$

Case (i): For $\eta=2.5$ and $b=0.5$, the effective bending modulus plot shown in figure $10(b)$ has soft sections of low bending modulus in the corners of the super-elliptical membrane $\left(34^{\circ}<\theta<56^{\circ}\right)$, where the fibers oriented in helical mode does not influence the membrane elasticity and stiffened sections of high bending modulus on the sides $\left(\theta=0,90^{\circ}\right)$ where the fibers are oriented in ring mode contribute to strengthening of the membrane. This bending stiffness variation is compatible with growth and inflation with invariant shape, since the softer corners can change curvature (increase its radius) but the stiffer and more straight sides remain unchanged. 
Case (ii): For $\eta=6$ and $b=3.5$, the effective bending modulus plot shown in figure 10(c) has soft sections of low bending modulus in the sides $\left(0^{\circ}<\theta<32^{\circ}\right.$ and $\left.58^{\circ}<\theta<90^{\circ}\right)$, where the fibers oriented in ring mode promotes elastic softening of the membrane as discussed in section 3.4 and stiffened sections of high bending modulus in the corners $\left(32^{\circ}<\theta<58^{\circ}\right)$, where the fibers oriented in ring mode contribute to strengthening of the membrane. This bending stiffness variation is incompatible with growth and inflation with invariant shape and the initial super-ellipse can only grow distortions of the sides. Case (iii): For $\eta=4$ and $b=0.75$, the effective bending modulus plot shown in figure $10(\mathrm{~d})$ has weakened sections of low bending modulus in the sides $\left(0^{\circ}<\theta<13^{\circ}\right.$ and $77^{\circ}<\theta<90^{\circ}$ ), where the fibers oriented in ring mode promotes elastic softening of the membrane, and the corners of the super-elliptical membrane $\left(37^{\circ}<\theta<53^{\circ}\right)$, where the fibers oriented in helical mode does not influence the membrane elasticity, and stiffened sections of high bending modulus in the regions between the sides and the corners $\left(13^{\circ}<\theta<37^{\circ}\right.$ and $\left.53^{\circ}<\theta<77^{\circ}\right)$ where the fibers oriented in ring mode contribute to strengthening of the membrane. Growth and expansion produce maximal shape change since the contour now has two weakened sections.

A schematic representing the shape of the fiber-laden membranes resulting from deformation of membranes shown in figure 10(a) is presented in figure 11.
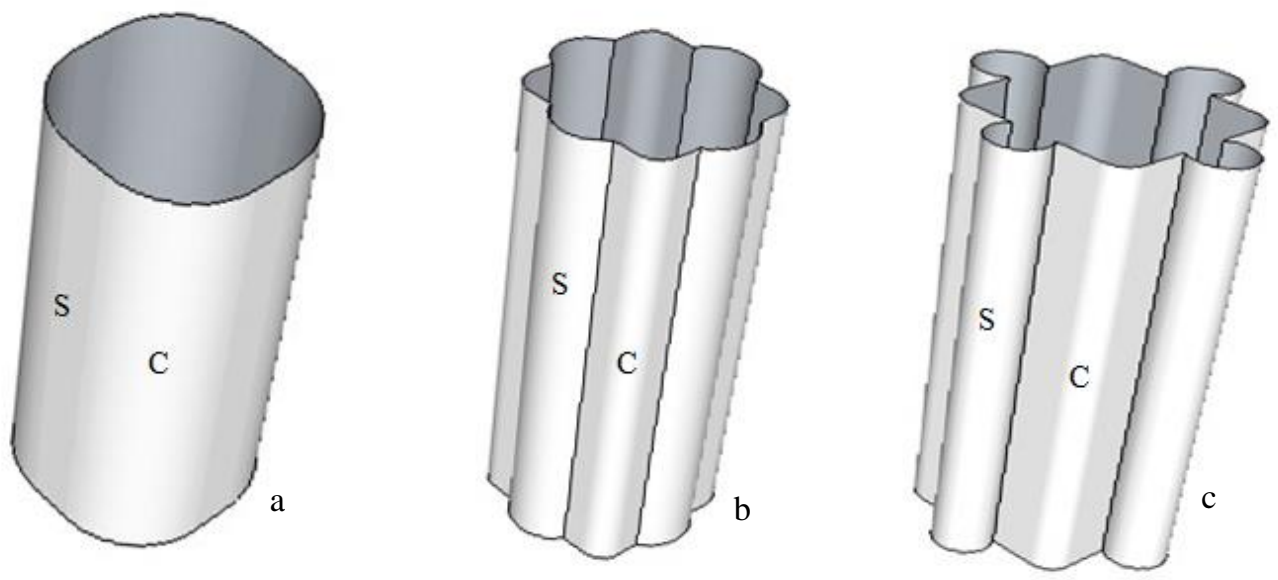
Figure 11. Shape of the fiber-laden membranes resulting from expansion of super-elliptical membrane: (a) $\eta=2.5$ and $b=0.5$ leads to shape invariant growth, (b) $\eta=6$ and $b=3.5$ leads to growth with weak shape change, (c)) $\eta=4$ and $\mathrm{b}=0.75$ leads to growth with large shape change. Stiff sections between sides and corners lead to large shape changes. $\mathrm{S}$ - side, $\mathrm{C}$ - corner

Figure 11 shows that fiber-driven stiffness regulation results in three characteristic growth modes in super-ellipses: (i) shape invariant growth (stiff sides) (Figure 11(a)), (ii) growth with weak shape change (stiff corners) (Figure 11(b)), and (iii) growth with strong shape change (stiff sections between the sides and the corners) (Figure 11(c)).

\section{Conclusions}

In this work, the curvature-induced cellulose microfibril orientation in plant cell walls at dilute concentrations is investigated by solving numerically an integrated micromechanical model developed for fiber-laden membranes. The model was formulated using a Helmholtz free energy that integrates the Helfrich bending/torsion membrane energy (eqn.7), the nematic Landau-de Gennes fiber orientation energy (eqn.8), and competing curvophilic and curvophobic interactions between the membrane and microfibrils (eqn.7). The influence of membrane curvature on the fiber structure in cylindrical membranes of circular cross-section was characterized (Figure 2), showing that as the radius increases a sequence of line, helical, and ring fiber orientation modes emerge. The fiber order is maximum at low membrane radius and asymptotically vanishes to zero as the membrane grows infinitely large.

The model predictions are validated by comparing the predicted microfibril orientation (figure 2) with experimentally observed orientation in cell wall of tracheids ${ }^{48}$ (figure 3(a)). The primary cell walls deposited during growth of plant cell from a small radius to a large radius, show progressive change in fiber orientation from line mode to ring mode (figure 3(a)-1 \& 3(a)-2). The 
deposition of secondary cell wall layers results in decrease of membrane radius and consequently helical and line modes are observed in $S_{1}$ and $S_{2}$ layers, respectively (figure 3(a)-3). The discrepancy between the observed helical or cross helical mode and predicted line mode in $\mathrm{S}_{3}$ layer might be resolved by modeling the temporal dynamics of fiber orientation.

The role of fiber structure on the elasticity of the fiber-laden membranes is investigated by analyzing the effective bending modulus of the system as a function of dimensionless radius (Figure 4). The analysis shows that the bending elasticity of the fiber-laden membranes is not influenced by the presence of fibers oriented in line and helical mode irrespective of the magnitude of the fiber order, while the fibers oriented in ring mode can either strengthen or weaken the membrane. The role of fibers oriented in ring mode on membrane bending elasticity is influenced by the magnitude of fiber order and is a result of the interplay between pure membrane elasticity, curvophobic, and curvophilic interactions. The maximum stiffening occurs when the membrane radius $\mathrm{R}$ is nearly equal to the internal length scale $\ell_{\mathrm{o}}$ (Figure 5 ).

With the aim of characterizing graded fibrous structures, the model was then extended to cylindrical membranes of super-elliptical cross-section in which the membrane curvature varies smoothly around the circumference of the membrane. The cross-sectional shape of the membranes is represented by Lame' curves. The effect of varying the shape and size parameter of Lame' curves on the fiber structure were investigated. Multimodal fiber orientations resulting in graded fiber orientation may arise both in radial and azimuthal directions due to curvature gradients between the sides and the corners induced by the shape of the plant cells and change in its size during cell growth and secondary cell wall deposition (Figure $6 \& 7$ ). This prediction is supported by the observation that the fiber orientation angle in the corners measured with respect to azimuthal coordinate $(\alpha)$ is $15^{\circ}$ to $25^{\circ}$ greater than in the sides ${ }^{14}$. 
Phase maps of fiber orientation modes and uniformity of fiber orientation distribution were developed to gain insight on the effect of membrane geometry on fiber structure in fiber-laden membranes (Figure $8 \& 9$ ). Assuming that the mechanics of synthetic composites apply to plant cell walls ${ }^{\mathbf{5 2 5 3}}$, the phase maps indicate a trade off between microcracking resistance and strength in plant cell walls of super-elliptical cross-section, but further plant-based experimental evidence is necessary. The super-elliptical membranes have a spatially inhomogeneous effective bending modulus (figure 10), which lead to different growth modes. Three distinct spatial inhomogeneity patterns in effective bending modulus of membranes and their characteristic growth modes are analyzed (figure 11). The shape invariant growth through membrane stretching predominant in plant cell walls are predicted to arise when corners sections have low bending modulus and the sides have stiffened sections of high bending modulus.

The coupling between in-plane fiber orientation and order and the curvature of the membranes demonstrated by plant cell walls in nature has the potential to open up a novel venue to control two dimensional anisotropic soft matter with tailored functionalities ${ }^{\mathbf{5 6}}$.

\section{Acknowledgements}

Financial support for this research was provided by Le Fonds Québécois de la Recherche sur la Nature et les Technologies (FQNRT). (A.D.R \& D.P). Y.K.M. is the recipient of post-graduate scholarships from McGill Engineering Doctoral Awards (MEDA) and the Eugenie Ulmer Lamothe fund of the Department of Chemical Engineering of McGill University.

\section{Appendix A}

Geometric characterization of a curved surface 
The purpose of this Appendix is to derive the average $\mathrm{H}$ and deviatoric $\mathrm{D}$ membrane curvatures used throughout the paper.

The geometry of an arbitrarily curved surface is characterized by the mean surface curvature $\mathrm{H}$ and the deviatoric curvature $\mathrm{D}$ given by ${ }^{27,28}$

$$
\mathrm{H}=-\frac{1}{2} \nabla_{\mathrm{s}} \cdot \mathbf{k}=\frac{1}{2} \mathbf{b}: \mathbf{I}_{\mathrm{s}}=-\frac{1}{2} \mathbf{I}_{\mathrm{s}}: \nabla_{\mathrm{s}} \mathbf{k}=\frac{1}{2}\left(v_{1}+\mathrm{v}_{2}\right), \mathrm{D}=\frac{1}{2} \mathbf{b}: \mathbf{q}=\frac{1}{2}\left(v_{1}-\mathrm{v}_{2}\right)
$$

where $\nabla_{\mathrm{s}}=\mathbf{I}_{s} \cdot \nabla$ is the surface gradient, $\mathbf{I}_{s}=\mathbf{I}-\mathbf{k k}$ is the $2 \times 2$ unit surface dyadic, $\mathbf{k}$ is the unit normal, $\mathbf{I}$ is the $3 \times 3$ unit dyadic, $\mathbf{b}$ is the $2 \times 2$ symmetric surface curvature dyadic, and $\left\{v_{\mathrm{m}}, \mathbf{e}_{\mathrm{m}}\right\}$, $m=1,2$ are the eigenvalues and eigenvectors of $\mathbf{b}$. The principal curvatures $\left(v_{1}, v_{2}\right)$ define the principal radii of curvature $\left(r_{m}\right)$ of the surface: $v_{m}=-1 / r_{m}$. The magnitude of the deviatoric curvature $\mathrm{D}$ is a useful non-sphericity index, since for a sphere $\mathrm{D}=0$. For a cylindrical surface, the average surface curvature $\mathrm{H}$ and deviatoric curvature $\mathrm{D}$ are equal.

The four independent basis surface tensors are ${ }^{\mathbf{1 , 5 7}}$ :

$$
\left\{\mathbf{I}_{s}, \mathbf{q}, \boldsymbol{\varepsilon}_{s}, \mathbf{q}_{1}=\mathbf{q} \cdot \boldsymbol{\varepsilon}_{s}\right\}
$$

The matrix representations of the basis vectors in the principal frame are ${ }^{\mathbf{3 4 , 5 8}}$.

$$
\mathbf{I}_{s}=\left(\begin{array}{ll}
1 & 0 \\
0 & 1
\end{array}\right), \mathbf{q}=\left(\begin{array}{cc}
1 & 0 \\
0 & -1
\end{array}\right), \boldsymbol{\varepsilon}_{s}=\left(\begin{array}{cc}
0 & 1 \\
-1 & 0
\end{array}\right), \mathbf{q}_{1}=\mathbf{q} \cdot \boldsymbol{\varepsilon}_{s}=\left(\begin{array}{ll}
0 & 1 \\
1 & 0
\end{array}\right)
$$

where $\boldsymbol{\varepsilon}_{\mathrm{s}}$ is the surface alternator tensor. The tensor basis orthonormality yields the following results:

$$
\begin{aligned}
& \mathbf{I}_{s}: \mathbf{I}_{s}=\mathbf{q}: \mathbf{q}=\boldsymbol{\varepsilon}_{s}: \boldsymbol{\varepsilon}_{s}=\mathbf{q}_{1}: \mathbf{q}_{1}=2, \mathbf{I}_{s}: \mathbf{q}=\mathbf{I}_{s}: \boldsymbol{\varepsilon}_{s}=\mathbf{I}_{s}: \mathbf{q}_{1}=\mathbf{q}: \boldsymbol{\varepsilon}_{s}=\mathbf{q}: \mathbf{q}_{1}=\boldsymbol{\varepsilon}_{s}: \mathbf{q}_{1}=0, \\
& \mathbf{I}_{s} \cdot \mathbf{q}=\mathbf{q}, \mathbf{I}_{s} \cdot \mathbf{q}_{1}=\mathbf{q}_{1}, \mathbf{I}_{s} \cdot \boldsymbol{\varepsilon}_{s}=\boldsymbol{\varepsilon}_{s}, \mathbf{q} \cdot \mathbf{q}_{1}=\boldsymbol{\varepsilon}_{s}, \mathbf{q} \cdot \boldsymbol{\varepsilon}_{s}=\mathbf{q}_{1}, \mathbf{q}_{1} \cdot \boldsymbol{\varepsilon}_{s}=\mathbf{q}
\end{aligned}
$$

Any $2 \times 2$ tensor $\mathbf{Z}$ can be expanded as: 
$\mathbf{Z}=\underbrace{\frac{1}{2}\left(\mathbf{Z}: \mathbf{I}_{s}\right) \mathbf{I}_{s}}_{\text {trace }}+\underbrace{\frac{1}{2}(\mathbf{Z}: \mathbf{q}) \mathbf{q}}_{\text {diagonal traceless }}+\underbrace{\frac{1}{2}\left(\mathbf{Z}: \boldsymbol{\varepsilon}_{s}\right) \boldsymbol{\varepsilon}_{s}}_{\text {antisymmetric }}+\underbrace{\frac{1}{2}\left(\mathbf{Z}: \mathbf{q}_{1}\right) \mathbf{q}_{1}}_{\text {symmetric off-diagonal }}$

where the subtext identifies the nature of the tensor. A symmetric $2 \times 2$ tensor diagonal in the principal coordinate frame simplifies to:

$\mathbf{Z}=\frac{1}{2}\left(\mathbf{Z}: \mathbf{I}_{s}\right) \mathbf{I}_{s}+\frac{1}{2}(\mathbf{Z}: \mathbf{q}) \mathbf{q}$

Using eqn.(A.6), the curvature tensor $\mathbf{b}$ can be decomposed into a trace and a deviatoric curvature tensor: $\mathbf{b}=\mathrm{HI} \mathbf{s}_{\mathrm{s}}+\mathrm{Dq}$

\section{Appendix B}

\section{Free energy minimization of fiber-laden membrane and selected orientation modes}

The purpose of this Appendix is: (i) to derive the Helmholtz free energy density in terms of fiber angle $\alpha$, fiber order $S$, and membrane curvatures H/D, and (ii) to obtain the orientation and ordering modes that result from the free energy minimization. These results are used in Sections

\section{1 and 3.2}

Using the principal curvature frame $\left(\mathbf{e}_{1}, \mathbf{e}_{2}\right)$, parametrizing the director with $\mathbf{n}=(\cos \alpha, \sin \alpha)$, where $\alpha$ is the fiber orientation angle with respect to azimuthal coordinate of the cylindrical membrane $(\cos \alpha=$ n.e 1$)$ the free energy $\rho \hat{\mathrm{A}}_{\text {fiber }}+\rho \hat{\mathrm{A}}_{\text {coupling }}$ becomes:

$$
\begin{aligned}
& \rho \hat{A}_{\text {fiber }}+\rho \hat{A}_{\text {coupling }}=\beta_{o}+\beta_{2} \cos ^{2} \alpha+\beta_{4} \cos ^{4} \alpha \\
& \beta_{\mathrm{o}}=\left(\left(\mathrm{a}_{2}+\frac{\mathrm{a}_{5}}{2}\right)+\frac{\mathrm{a}_{3}}{2} \mathrm{H}\right) \mathrm{H}-\left(\left(\mathrm{a}_{2}+\mathrm{a}_{5}\right) \mathrm{D}+\mathrm{a}_{3} \mathrm{HD}\right) \mathrm{S}+\left(\frac{\mathrm{a}_{1}}{4}+\frac{\mathrm{a}_{5}}{2} \mathrm{H}+\frac{\mathrm{a}_{3}}{2} \mathrm{D}^{2}\right) \mathrm{S}^{2}+\frac{\mathrm{a}_{4}}{16} \mathrm{~S}^{4} \\
& \beta_{2}=2\left(\mathrm{a}_{2}+\mathrm{a}_{3} \mathrm{H}-\mathrm{a}_{3} \mathrm{DS}+\mathrm{a}_{5}\right) \mathrm{DS}, \beta_{4}=2 \mathrm{a}_{3} \mathrm{D}^{2} \mathrm{~S}^{2}
\end{aligned}
$$


This energy (eqn.(B.1)) vanishes for flat isotropic states: $\mathrm{D}=\mathrm{H}=\mathrm{S}=0$. The quartic contribution $\left(\beta_{4} \cos ^{4} \alpha\right)$ is necessary to observe fiber alignment away from the principal axes. The form of the free energy is identical to that describing the interfacial tension of nematic-substrate interfaces ${ }^{\mathbf{4 7}}$.

For a given geometry, the preferred fiber orientation $(\alpha)$ and fiber order $(S)$ is found by minimizing the Helmholtz free energy density $\rho \hat{A}$ :

$$
\begin{aligned}
& \rho \frac{\partial \hat{\mathrm{A}}}{\partial \mathrm{S}}=\frac{\mathrm{a}_{4}}{4} \mathrm{~S}^{3}+\left(\frac{\mathrm{a}_{1}}{2}+\mathrm{a}_{5} \mathrm{H}\right) \mathrm{S}+\left(\mathrm{a}_{2}+\mathrm{a}_{3} \mathrm{H}+\mathrm{a}_{5}+\mathrm{a}_{3} \mathrm{DS} \cos 2 \alpha\right) \mathrm{D} \cos 2 \alpha=0 \\
& \rho \frac{\partial \hat{\mathrm{A}}}{\partial \alpha}=\left(\frac{\beta_{2}}{2 \beta_{4}}+\cos ^{2} \alpha\right) \sin \alpha \cos \alpha=0
\end{aligned}
$$

where the second equation is re-written in a more revealing format. In terms of the fiber director, there are three equilibria states: (a) orientation along major curvature, $\alpha=0$; (b) along the minor curvature, $\alpha=\pi / 2$; and (c) oblique, $\cos ^{2} \alpha=-\beta_{2} / 2 \beta_{4}$, as follows.

(a) Orientation along the major curvature. Here $\mathbf{n}=\mathbf{e}_{1}$ and the scalar order parameter $\mathbf{S}$ satisfies a cubic:

$$
\alpha=0, \frac{a_{4}}{4} S^{3}+\left(\frac{a_{1}}{2}+a_{5} H+a_{3} D^{2}\right) S+\left(a_{2} D+a_{3} H D+a_{5} D\right)=0
$$

The necessary conditions are

$$
\frac{\beta_{2}}{2 \beta_{4}}=\frac{\left(a_{2}+a_{5}+a_{3}(H-D S)\right)}{2 a_{3} D S}>0, \quad \beta_{2}=2\left(a_{2}+a_{3} H-a_{3} D S+a_{5}\right) D S<0
$$

Since $\beta_{4}<0, \beta_{2}<0$ the minimum energy corresponds to orientation along the largest principal curvature $(\alpha=0)$. For a cylinder, this is the azimuthal direction.

(b) Orientation along the minor curvature. Here $\mathbf{n}=\mathbf{e}_{2}$ and the scalar order parameter $\mathbf{S}$ satisfies a cubic: 
$\alpha=\pi / 2, \frac{a_{4}}{4} S^{3}+\left(\frac{a_{1}}{2}+a_{5} H+a_{3} D^{2}\right) S-\left(a_{2} D+a_{3} H D+a_{5} D\right)=0$

The necessary conditions are

$\frac{\beta_{2}}{2 \beta_{4}}=\frac{\left(a_{2}+a_{5}+a_{3}(H-D S)\right)}{2 a_{3} D S}>0, \quad \beta_{2}=2\left(a_{2}+a_{3} H-a_{3} D S+a_{5}\right) D S>0$

Since $\beta_{4}>0, \beta_{2}>0$ the minimum energy corresponds to the orientation along the smallest principal curvature $(\alpha=\pi / 2)$. For a cylinder this is the axial direction.

(c) Oblique orientation along the minor curvature. Here $\mathbf{n}=\mathbf{e}_{2}$ and the scalar order parameter $\mathbf{S}$ satisfies a cubic:

$\cos ^{2} \alpha=-\frac{\beta_{2}}{2 \beta_{4}}=-\frac{\left(a_{2}+a_{5}+a_{3} H-a_{3} D S\right)}{2 a_{3} D S},\left(\frac{a_{1}}{2}+a_{5} H\right) S+\frac{a_{4}}{4} S^{3}=0$

The necessary conditions are $-2 \beta_{4}<\beta_{2}<0, \beta_{4}>0$

The ordering under oblique conditions is the result of a competition between the Landau-de Gennes terms $\left(\mathrm{a}_{1}, \mathrm{a}_{2}\right)$ and the curvophilic $\mathrm{a}_{5}$ term.

Next, we discuss transitions and assume without loss of generality that all the energy coefficients are positive $\left\{a_{i}\right\}>0$. For $\beta_{4}>0$, orientation transitions from oblique to principal curvature directions occurs for $\mathrm{D} \neq 0$ as follows. (a) Oblique-major curvature (e $\left.\mathbf{e}_{1}\right)$ transition: as $-\beta_{2} \rightarrow 2 \beta_{4}, \alpha \rightarrow 0$. In this case $\beta_{2}$ becomes sufficiently negative to balance $\beta_{4}$. At the transition when $\alpha \rightarrow 0$ the curvatures obey:

$\mathrm{D} \sqrt{-\frac{\left(2 \mathrm{a}_{1}+4 \mathrm{a}_{5} \mathrm{H}\right)}{\mathrm{a}_{4}}}+\mathrm{H}=-\frac{\left(\mathrm{a}_{2}+\mathrm{a}_{5}\right)}{\mathrm{a}_{3}}<0$

This transition will occur at sufficiently small curvatures so that the effect of $a_{2}$ and as prevail (see discussion below eqn.(B.10)). 
(b) Oblique-minor curvature $\left(\mathbf{e}_{2}\right)$ : as $\beta_{2} \rightarrow 0, \alpha \rightarrow \pi / 2$. This transition to orientation along the minimum curvature occurs as the tension introducing $\cos ^{2} \alpha$ in the free energy vanishes (see eqn.(B.1c)) and this can happen only at sufficiently large H and D. At the transition the curvatures obey:

$H-D \sqrt{-\frac{\left(2 a_{1}+4 a_{5} H\right)}{a_{4}}}=-\frac{\left(a_{2}+a_{5}\right)}{a_{3}}$

Next we demonstrate an application of these results (eqns.(B.9, B.10) for the case of a cylinder of radius $\mathrm{R}$, when $\mathrm{D}=\mathrm{H}=-(1 / 2 \mathrm{R})$, relevant to plant cell walls of cylindrical cells with circular cross-section. Using (eqns.(B.9, B.10), we find that the critical transition radii $\mathrm{R}_{1}$ and $\mathrm{R}_{2}$ for oblique/major curvature and oblique/minor curvature, respectively, obey:

$\mathrm{R}_{1}=\frac{\mathrm{a}_{3}}{2\left(\mathrm{a}_{2}+\mathrm{a}_{5}\right)}\left\{1+\sqrt{-\frac{2\left(\mathrm{a}_{1}-\frac{\mathrm{a}_{5}}{\mathrm{R}_{1}}\right)}{\mathrm{a}_{4}}}\right\}=\frac{\mathrm{a}_{3}}{2\left(\mathrm{a}_{2}+\mathrm{a}_{5}\right)}(1+\mathrm{S})$
$\mathrm{R}_{2}=\frac{\mathrm{a}_{3}}{2\left(\mathrm{a}_{2}+\mathrm{a}_{5}\right)}\left\{1-\sqrt{-\frac{2\left(\mathrm{a}_{1}-\frac{\mathrm{a}_{5}}{\mathrm{R}_{2}}\right)}{\mathrm{a}_{4}}}\right\}=\frac{\mathrm{a}_{3}}{2\left(\mathrm{a}_{2}+\mathrm{a}_{5}\right)}(1-\mathrm{S})=<\mathrm{R}_{1}$

where we used eqn.(B.7b) for S. Figure 1 shows the stability regions for line, helix and ring modes as a function of the radius for a cylindrical membrane. A small cylinder radius induces axial orientation while larger radius induces azimuthal orientation, and the interval over which the intermediate oblique state exists is: 


$$
\mathrm{R}_{1}-\mathrm{R}_{2}=\frac{\mathrm{a}_{3}}{2\left(\mathrm{a}_{2}+\mathrm{a}_{5}\right)}\left(\sqrt{\frac{\left(\frac{\mathrm{a}_{5}}{\mathrm{R}_{1}}-\mathrm{a}_{1}\right)}{\mathrm{a}_{4} / 2}}+\sqrt{\frac{\left(\frac{\mathrm{a}_{5}}{\mathrm{R}_{2}}-\mathrm{a}_{1}\right)}{\mathrm{a}_{4} / 2}}\right)
$$

\section{Appendix C}

\section{Effective Bending Modulus of a fiber-laden membrane}

The purpose of this Appendix is: (i) to derive the effective fiber modulus of a fiber laden membrane in terms of fiber angle $\alpha$, fiber order $\mathrm{S}$, and membrane curvatures $\mathrm{H}$, and (ii) to establish the relations between bending stiffness and fiber orientation and order. These results are used in Section 3.4

The effective bending modulus of an arbitrarily curved fiber-laden membranes $\mathrm{k}_{\mathrm{c}, \text { eff }}$ has contributions due to membrane bending elasticity, $\mathrm{k}_{\mathrm{c}, \text { membrane, }}$ and membrane curvature induced nematic ordering of fibers, $\mathrm{k}_{\mathrm{c}, \text { fiber }}$. The fiber contribution to effective bending modulus, $\mathrm{k}_{\mathrm{c}, \mathrm{fiber}}$ is given by the second derivative of the fiber and coupling free energies $\rho \hat{\mathrm{A}}_{\text {fiber }}+\rho \hat{\mathrm{A}}_{\text {coupling }}$ with respect to mean surface curvature $\mathrm{H}$ :

$$
\begin{aligned}
\mathrm{k}_{\mathrm{c}, \text { fiber }} & =\frac{\mathrm{d}^{2}\left(\rho \hat{\mathrm{A}}_{\text {fiber }}+\rho \hat{\mathrm{A}}_{\text {coupling }}\right)}{\mathrm{dH} \mathrm{H}^{2}}=\left\{1-2 \mathrm{~S}+\mathrm{S}^{2}+4 \mathrm{~S} \cos ^{2} \alpha-4 \mathrm{~S}^{2} \cos ^{2} \alpha+4 \mathrm{~S}^{2} \cos ^{4} \alpha\right\} \mathrm{a}_{3} \\
& +\left\{\left(\mathrm{a}_{2}+\mathrm{a}_{5}+2 \mathrm{a}_{3} \mathrm{H}-2 \mathrm{a}_{3} \mathrm{HS}+4 \mathrm{a}_{3} \mathrm{HS} \cos ^{2} \alpha\right) 4 \mathrm{~S} \sin \alpha\right\} \frac{\partial \cos \alpha}{\partial \mathrm{H}} \\
& +\left\{\left(\mathrm{a}_{2}+\mathrm{a}_{5}+2 \mathrm{a}_{3} \mathrm{H}-4 \mathrm{a}_{3} \mathrm{HS}\right) 2 \cos ^{2} \alpha-\left(\mathrm{a}_{2}+\mathrm{a}_{5}+2 \mathrm{a}_{3} \mathrm{H}\right)+\left(\mathrm{a}_{5}+2 \mathrm{a}_{3} \mathrm{H}\right) \mathrm{S}+8 \mathrm{a}_{3} \mathrm{HS} \cos ^{4} \alpha\right\} \frac{\partial \mathrm{S}}{\partial \mathrm{H}}
\end{aligned}
$$

The three fiber contributions to bending stiffness include the bare curvophobic effect $\left(a_{3}\right)$, the director $\operatorname{effect}(\partial \alpha / \partial \mathrm{H})$, and the order parameter $\operatorname{effect}(\partial \mathrm{S} / \partial \mathrm{H})$. As shown below maximum 
fiber reinforcing effect is obtained as expected when $\alpha=0$ and order is high. For $0<\mathrm{R}^{*}<0.1847$, the fibers are oriented in line mode and eqn.(C.1) simplifies to:

$\left.\mathrm{k}_{\mathrm{c}, \text { fiber }}\right|_{0<\mathrm{R}^{*}<0.1847}=\left(1-2 \mathrm{~S}+\mathrm{S}^{2}\right) \mathrm{a}_{3}+\left(2 \mathrm{a}_{3} \mathrm{HS}-2 \mathrm{a}_{3} \mathrm{H}-\mathrm{a}_{2}-\mathrm{a}_{5}+\mathrm{a}_{5} \mathrm{~S}\right) \frac{\partial \mathrm{S}}{\partial \mathrm{H}}$

For $\mathrm{R}^{*}>0.3867$, the fibers are oriented in ring mode and eqn.(C.1) simplifies to:

$\left.\mathrm{k}_{\mathrm{c}, \text { fiber }}\right|_{\mathrm{R}^{*}>0.3867}=\left(1+2 \mathrm{~S}+\mathrm{S}^{2}\right) \mathrm{a}_{3}+\left(2 \mathrm{a}_{3} \mathrm{HS}+2 \mathrm{a}_{3} \mathrm{H}+\mathrm{a}_{2}+\mathrm{a}_{5}+\mathrm{a}_{5} \mathrm{~S}\right) \frac{\partial \mathrm{S}}{\partial \mathrm{H}}$

The derivates $\partial \mathrm{S} / \partial \mathrm{H}, \partial(\cos \alpha) / \partial \mathrm{H}$ in eqn. (C.1), (C.2), and (C.3) are evaluated numerically using central difference method at each value of $\mathrm{R}^{*}$. The membrane contribution to effective bending

modulus, $\mathrm{k}_{\mathrm{c}, \text { membrane }}$ is given by the second derivative of the membrane free energy $\rho \hat{\mathrm{A}}_{\text {membrane }}$ with respect to mean surface curvature $\mathrm{H}$ :

$\mathrm{k}_{\mathrm{c}, \text { membrane }}=\frac{\mathrm{d}^{2}\left(\rho \hat{\mathrm{A}}_{\text {membrane }}\right)}{\mathrm{dH}^{2}}=4 \mathrm{k}_{\mathrm{c}}$

In this work, the dimensionless membrane bending modulus $\mathrm{k}_{\mathrm{c}}$ is set equal to 1 representing similar magnitudes of membrane elasticity and membrane-fiber interactions.

\section{References}

1. A. D. Rey, Soft Matter, 2007, 3, 1349-1368.

2. T. Rasing and I. Muševič, Surfaces and interfaces of liquid crystals, Springer Verlag, Berlin, 2004.

3. S. Martellucci, A. Chester and A. Mignani, Optical Sensors and Microsystems, SpringerVerlag, 2000.

4. H. Liang, S. Schymura and J. Lagerwall, Conference book 38th Topical Meeting on Liquid Crystals, Mainz, Germany, 2010. 
5. J. Klett, Cellular ceramics: structure, manufacturing, properties and applications, 2005, 2, 137.

6. I. Burgert, American Journal of Botany, 2006, 93, 1391.

7. M. Tang and M. Hong, Molecular BioSystems, 2009, 5, 317-322.

8. J. Zimmerberg and M. Kozlov, Nature Reviews Molecular Cell Biology, 2005, 8, 9-19.

9. C. Brett, K. Waldron and K. Waldron, Physiology and biochemistry of plant cell walls, Springer, 1996.

10. I. Burgert, M. Eder, N. Gierlinger and P. Fratzl, Planta, 2007, 226, 981-987.

11. A. M. C. Emons and B. M. Mulder, Trends in plant science, 2000, 5, 35-40.

12. J. Rose, The plant cell wall, CRC Press, 2003.

13. J. Barnett and V. Bonham, Biological reviews, 2004, 79, 461-472.

14. S. Wainwright, Mechanical design in organisms, Princeton Univ Pr, 1982.

15. A. Neville, Biology of fibrous composites: development beyond the cell membrane, Cambridge Univ Pr, 1993.

16. E. Belamie, G. Mosser, F. Gobeaux and M. Giraud-Guille, Journal of Physics: Condensed Matter, 2006, 18, S115.

17. S. C. Cowin, Journal of Non-Newtonian Fluid Mechanics, 2004, 119, 155-162.

18. J. Revol, H. Bradford, J. Giasson, R. Marchessault, and D. Gray, International. Journal of Biological Macromolecules, 1992, 14, 170-172.

19. J. Revol, L. Godbout, X. Dong, D. Gray, H. Chanzy, G. Maret, Liquid Crystals, 1994, 16, $127-134$.

20. A. D. Rey, Soft Matter, 2010, 6, 3402-3429.

21. Y. Murugesan and A. Rey, Polymers, 2010, 2, 766-785. 
22. J. Roland, D. Reis, M. Mosiniak and B. Vian, Journal of Cell Science, 1982, 56, 303-318.

23. M. Fošnaric, K. Bohinc, D. Gauger, A. Iglic, V. Kralj-Iglic and S. May, J. Chem. Inf. Model, 2005, 45, 1652-1661.

24. A. D. Rey and Y. K. Murugesan, Continuum Mechanics and Thermodynamics, 2010, 23, 45-61.

25. Y. Murugesan and A. Rey, Macromolecular Theory and Simulations, 2010, 19, 113-126.

26. Y. Murugesan and A. Rey, Journal of Non-Newtonian Fluid Mechanics, 2010, 165, 32-44.

27. A. Goetz, Introduction to differential geometry, Addison-Wesley Reading, MA, 1970.

28. M. Do Carmo, Prentice-Hall, Englewood Cliffs, NJ, 1976.

29. P. Almeida and F. Wiegel, Journal of theoretical biology, 2006, 238, 269-278.

30. R. Mezzenga, J. Jung and J. Adamcik, Langmuir, 2010, 26, 10401-10405.

31. J. Jung and R. Mezzenga, Langmuir, 2010, 26, 504-514.

32. P. Kralchevsky and K. Nagayama, Particles at fluid interfaces and membranes, Elsevier, Amsterdam, 2001.

33. S. Ljunggren, J. Eriksson and P. Kralchevsky, J Colloid Interface Sci, 1997, 191, 424-441.

34. R. Rosso and E. Virga, Modeling of Biological Materials, 2007, 323-357.

35. S. Desrochers, D. Pasini and J. Angeles, Journal of Mechanical Design, 2010, 132, 041011.

36. D. Pasini, International Journal of Mechanics and Materials in Design, 2009, 5, 175-193.

37. D.Pasini, International Journal of Design \& Nature and Ecodynamics, 2008, 3, 1-26

38. P. de Gennes and J. Prost, The physics of liquid crystals, Oxford University Press, USA, 1995.

39. J. C. Eriksson and S. Ljunggren, Surfactant science series, 2004, 119, 547-614. 
40. A. D. Rey, Langmuir, 2006, 22, 219-228.

41. P. Biscari and E. Terentjev, Physical Review E, 2006, 73, 51706.

42. J. Fournier and P. Galatola, Physical Review E, 1999, 60, 2404-2407.

43. Q. Liu, Y. Liu, B. Hu and Z. Ou-Yang, Physics Letters A, 2006, 352, 358-361.

44. W. Sung and S. Lee, Europhysics Letters, 2004, 68, 596.

45. E. P. Lewandowski, P. C. Searson and K. J. Stebe, J. Phys. Chem. B, 2006, 110, 42834290.

46. E. P. Lewandowski, M. Cavallaro Jr, L. Botto, J. C. Bernate, V. Garbin and K. J. Stebe, Langmuir, 2010, 26, 15142-15154.

47. S. Faetti, in The Physics of Liquid Crystalline Materials, eds. I. C. Khoo and F. Simoni, Gordon and Breach, New York, 1991.

48. H. Abe and R. Funada, IAWA J, 2005, 26, 161-174.

49. A. Wardrop, Australian Journal of Botany, 1958, 6, 299-305.

50. C. Anderson, A. Carroll, L. Akhmetova and C. Somerville, Plant physiology, 2010, 152, 787-796.

51. E. K. H. Salje, Phase transitions in ferroelastic and co-elastic crystals: an introduction for mineralogists, material scientists, and physicists, Cambridge University Press, Cambridge, UK, 1993.

52. A. Giannakopoulos, Thin Solid Films, 1998, 332, 172-179.

53. C. H. Chen and C. H. Cheng, International Journal of Solids and Structures, 1996, 33, 2519-2539.

54. R. R. Netz and P. Pincus, Physical Review E, 1995, 52, 4114-4128. 
55. E. Katifori, S. Alben, E. Cerda, D. R. Nelson and J. Dumais, Proceedings of the National Academy of Sciences, 2010, 107, 7635.

56. A. Turner, V. Vitelli and D. Nelson, Reviews of Modern Physics, 2010, 82, 1301-1348.

57. A. D. Rey, Journal of colloid and interface science, 2006, 304, 226-238.

58. A. D. Rey, Rheologica Acta, 2008, 47, 861-871. 